\title{
Racemisierungsfreie Substitution von 2-(Sulfonyloxy)carbonsäureestern mit Sauerstoff- und Schwefelnucleophilen
}

\author{
Ulrike Burkard ${ }^{1)}$ und Franz Effenberger* \\ Institut für Organische Chemie der Universität Stuttgart, \\ Pfaffenwaldring 55, D-7000 Stuttgart 80
}

Eingegangen am 14. Oktober 1985

Die 2-(Sulfonyloxy)propionsäureester $(S)-1$ a - c reagieren mit aus 2 gebildeten Phenolaten bzw. mit Carboxylaten 8 unter Inversion zu den 2-(Aryloxy)- (R)-3 bzw. 2-(Acyloxy)propionsäureestern $(R)-9$. Die durch die große Austrittstendenz des Trifluormethansulfonats bedingte hohe Reaktivität von (S)-1 a führt, im Vergleich zu den Umsetzungen mit (S)-1 b bzw. (S)-1 c, unter milderen Reaktionsbedingungen zu besseren Ausbeuten an Substitutionsprodukten. Von den Äpfel- bzw. Bernsteinsäurederivaten 10a, 10 b bzw. 10d läßt sich lediglich das Trifluormethansulfonat $(S)-10$ a mit Carboxylaten bei tiefer Temp. $\left(-45^{\circ} \mathrm{C}\right)$ unter Konfigurationsumkehr zu entsprechenden Acyloxyverbindungen $(R)-12$ umsetzen, während das Mesylat $10 \mathrm{~b}$ und die Br-Verbindung 10d ausschließlich Eliminierung zu 11 geben. Aus 17 gebildete Mercaptide und Thiophenolate reagieren mit (S)-1 a-c ebenfalls unter Substitution und Konfigurationsumkehr; mit Kaliumrhodanid entsteht fast ausschließlich das Thiocyanat 19 neben nur Spuren von Isothiocyanat 20.

\section{Substitution of 2-(Sulfonyloxy)carboxylates with Oxygene and Sulfur Nucleophiles without Racemization}

The ethyl 2-(sulfonyloxy)propionates $(S)-1 \mathrm{a}-\mathrm{c}$ react with phenolates formed from 2 and with carboxylates 8 to give the respective 2-(aryloxy)- $(R)-3$ and 2-(acyloxy)propionates $(R)-9$ with inversion of configuration. Due to the high leaving tendency of the triflate group, (S)-1 a generally give higher yields of substitution products under milder conditions than the corresponding mesylate $(S)-1 \mathrm{~b}$ and tosylate $(S)-1 \mathrm{c}$. In the case of the reaction of malic and succinic acid derivatives only the triflate $(S)-10 \mathrm{a}$ is converted to the acyloxy compounds (R)-12 with carboxylates 8 at low temperature $\left(-45^{\circ} \mathrm{C}\right)$; with the mesylates $10 \mathrm{~b}$ and the bromide $10 \mathrm{~d}$ only elimination is observed. Mercaptides and thiophenolates formed from 17 react with $(S)-1 \mathrm{a}-\mathrm{c}$ analogously. With potassium thiocyanate $1 \mathrm{a}, \mathrm{b}$ react almost exclusively to give the thiocyanate 19; only traces of the corresponding isothiocyanate 20 are obtained.

In vorangegangenen Publikationen ${ }^{2)}$ haben wir über die Vorteile von Trifluormethansulfonat als Austrittsgruppe im Vergleich zu entsprechenden Halogeniden, Methansulfonaten oder Arylsulfonaten bei racemisierungsfreien Substitutionen optisch aktiver 2-substituierter Carbonsäureester berichtet. Trifluormethansulfonate sind - besonders bei den leicht zu Eliminierungen neigenden 2 -substituierten Derivaten von $\beta$-Phenylmilchsäure, Äpfelsäure und $\alpha$-Hydroxyglutarsäure - allen anderen Austrittsgruppen aufgrund ihrer hervorragenden Austrittstendenz ${ }^{2 \mathrm{a})}$ und wegen der geringen Nucleophilie des Triflat-Anions überlegen. 
Umsetzungen der Trifluormethansulfonate - insbesondere mit schwachen Nucleophilen sind daher unter wesentlich milderen Reaktionsbedingungen durchführbar, dabei wird einmal die Substitution gegenüber der Eliminierung begünstigt, zum anderen zeigen die erhaltenen Substitutionsprodukte eine deutlich höhere optische Reinheit als die ausgehend von entsprechenden Verbindungen mit anderen Austrittsgruppen ${ }^{2 a)}$.

Die enantioselektive Substitution 2-substituierter Carbonsäurederivate mit Schwefel- und Sauerstoffnucleophilen ist wegen der auf diese Weise zugänglichen biologisch aktiven Wirkstoffe von Interesse ${ }^{3,4)}$ und ermöglicht im Falle geeigneter Sauerstoffnucleophile eine Konfigurationsinversion von 2-Hydroxycarbonsäuren.

\section{Umsetzungen von 2-(Sulfonyloxy)propionsäureestern}

\section{a) Mit Phenolaten}

Für die als äußerst selektive Grasherbizide wirkenden 2-(Aryloxy)propionsäuren $^{4)}$ wurden enantioselektive Synthesen - ausgehend von (S)-MilchsäureDerivaten - entwickelt ${ }^{4}$. Dabei wurden hauptsächlich Verbindungen mit Methansulfonat und $p$-Tosylat als Austrittsgruppen verwendet, die bei der Umsetzung mit den stark nucleophilen Phenolaten zu Produkten mit hoher optischer Reinheit führten. Wir haben nun den von uns hergestellten $(S)$-2-(Trifluormethylsulfonyloxy)propionsäure-ethylester $[(S)-1 \text { a }]^{2 a)}$ sowie $(S)$-2-(Methylsulfonyloxy)- $[(S)-1$ b] und $(S)-2-(p$-Tolylsulfonyloxy)propionsäure-ethylester $[(S)-1 c]$ mit einigen Phenolaten unter $\mathrm{S}_{\mathrm{N}}$ 2-Bedingungen $\mathrm{zu}$ den $(R)$-2-(Aryloxy)propionsäure-ethylestern $(R)$-3 umgesetzt und die Reaktivitätsabstufungen der 2-substituierten Propionsäurederivate $(S)$-1 ermittelt (Tab. 1).

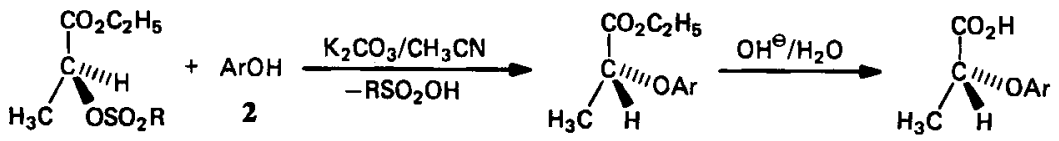

$$
\begin{aligned}
& \text { (S) }-1 \\
& \text { (R)-3 } \\
& (R)-4 \\
& \begin{array}{c|ccc}
(S)-1 & \text { a } & \text { b } & \text { c } \\
\hline R & \mathrm{CF}_{3} & \mathrm{CH}_{3} & \rho-\mathrm{CH}_{3} \mathrm{C}_{6} \mathrm{H}_{4}
\end{array} \\
& \begin{array}{r|cc}
(R)-3,4 & 2 & \text { b } \\
\hline \text { Ar } & 2,4-\mathrm{Cl}_{2} \mathrm{C}_{6} \mathrm{H}_{3} & 2,4,5-\mathrm{Cl}_{3} \mathrm{C}_{6} \mathrm{H}_{2}
\end{array} \\
& \text { rac }-\mathrm{H}_{3} \mathrm{C}-\mathrm{CHBr}-\mathrm{CO}_{2} \mathrm{C}_{2} \mathrm{H}_{5} \\
& \text { 1d } \\
& \begin{array}{r|cc}
(R)-3,4 & \text { c } & \text { d } \\
\hline \text { Ar } & 1-\mathrm{C}_{10} \mathrm{H}_{7} & 2-\mathrm{C}_{10} \mathrm{H}_{7}
\end{array}
\end{aligned}
$$

Die in Tab. 1 zusammengefaßten Ergebnisse zeigen, daß mit allen drei Alkylierungsmitteln $(S)$-1 a, b, c Verbindungen mit vergleichbarer optischer Reinheit erhalten werden, daß jedoch zur Erzielung hoher Produktausbeuten erwartungsgemäß mit $(S)$-1b und (S)-1 c deutlich härtere Reaktionsbedingungen als mit $(S)$ 1 a notwendig sind.

Die Ethylester $(R)-3$ lassen sich unter milden Bedingungen mit guten Ausbeuten zu den $(R)$-2-(Aryloxy)propionsäuren ( $R$ )-4 verseifen (Tab. 2). 
Tab. 1. (R)-2-(Aryloxy)propionsäure-ethylester $(R)-3$ aus (S)-2-(Sulfonyloxy)propionsäureethylestern (S)-1 und Phenolen 2a,b bzw. Naphtholen 2c, d

\begin{tabular}{|c|c|c|c|c|c|c|}
\hline \multirow{2}{*}{$(S)-1$} & \multirow[b]{2}{*}{2} & \multicolumn{2}{|c|}{ Reaktions- } & \multirow[b]{2}{*}{$\begin{array}{l}\text {-propionsäure- } \\
\text { ethylester }\end{array}$} & \multirow[b]{2}{*}{$\underset{\%}{\text { Ausb. }}$} & \multirow[b]{2}{*}{$\left(c=\begin{array}{c}{[\alpha]_{\mathrm{D}}^{2 \mathrm{D}}} \\
\left(c, \mathrm{CHCl}_{3}\right)\end{array}\right.$} \\
\hline & & Zeit & Temp. & & & \\
\hline$(S)-1 \mathbf{a}$ & $\begin{array}{l}\text { 2,4-Dichlor- } \\
\text { phenol (2a) }\end{array}$ & 3 & 20 & $\begin{array}{l}(R)-3 \mathbf{a} 2-(2,4- \\
\text { Dichlorphenoxy)- }\end{array}$ & 91 & $+28.4^{\circ}$ \\
\hline$(S)-1 b$ & $\mathbf{2 a}$ & 14 & 82 & $(R)-3 \mathbf{a}$ & 82 & $+28.7^{\circ}$ \\
\hline$(S)-1 c$ & $\mathbf{2 a}$ & 14 & 82 & $(R)-\mathbf{3} \mathbf{a}$ & 82 & $+27.9^{\circ}$ \\
\hline$(S)-1$ a & $\begin{array}{l}\text { 2,4,5-Trichlor- } \\
\text { phenol (2b) }\end{array}$ & 3 & 20 & $\begin{array}{l}(R)-3 \mathbf{b} 2-(2,4,5- \\
\text { Trichlorphenoxy)- }\end{array}$ & 93 & $+37.2^{\circ}$ \\
\hline$(S)-1 \mathfrak{a}$ & $\begin{array}{l}\alpha-\text { Naphthol } \\
\text { (2c) }\end{array}$ & 3 & 20 & $\begin{array}{l}(R)-3 c \\
\text { 2-(1-Naphthoxy)- }\end{array}$ & 84 & $\frac{-38.7^{\circ}}{\left(c=4, \mathrm{CHCl}_{3}\right)}$ \\
\hline$(S)-1 \mathbf{a}$ & $\begin{array}{l}\text { B-Naphthol } \\
\text { (2d) }\end{array}$ & 3 & 20 & $\begin{array}{l}(R)-3 d \\
\text { 2-(2-Naphthoxy)- }\end{array}$ & 90 & $\begin{array}{l}+83.5^{\circ} \\
\left(c=4.4, \mathrm{CHCl}_{3}\right) \\
+105.3^{\circ} \\
(c=2.5, \mathrm{EtOH})\end{array}$ \\
\hline
\end{tabular}

a) Zusammengeben der Edukte bei $0^{\circ} \mathrm{C}$.

Tab. 2. (R)-2-(Aryloxy)propionsäuren (R)-4 aus den Estern (R)-3 durch alkalische (A) bzw. saure Hydrolyse (B)

$(R)-3 \quad \begin{gathered}\text { Me- } \\ \text { thode }\end{gathered}$-propionsäure $\quad \begin{gathered}\text { Ausb. } \\ \%\end{gathered}$ Drehwert Lit.

\begin{tabular}{|c|c|c|c|c|c|}
\hline$(R)-\mathbf{3 a}$ & A & $\begin{array}{l}\text { 2-(2,4-Dichlor- } \\
\text { phenoxy)- }[(R)-4 \mathrm{a}]\end{array}$ & 85 & $\begin{array}{l}{[\alpha]_{D}^{25}=+26.9^{\circ}} \\
(c=4, \mathrm{EtOH})\end{array}$ & $\begin{array}{l}{[\alpha]_{\mathrm{D}}^{25}+28.1^{\circ}} \\
(\mathrm{EtOH})^{13)}\end{array}$ \\
\hline$(R)-\mathbf{3} \mathbf{a}$ & B & $(R)-4 a$ & 74 & & \\
\hline$(R)-3 b$ & A & $\begin{array}{l}2-(2,4,5-T \text { richlor- } \\
\text { phenoxy)- }[(R)-4 b]\end{array}$ & 81 & $\begin{array}{l}{[\alpha]_{\mathrm{D}}^{25}=+56.6^{\circ}} \\
(c=2, \text { Aceton })\end{array}$ & $\begin{array}{l}{[\alpha]_{\mathrm{D}}^{25}=+59.2^{\circ}} \\
\text { (Aceton) })^{14)}\end{array}$ \\
\hline$(R)-3 \mathrm{c}$ & $\mathbf{A}$ & $\begin{array}{l}\text { 2-(1-Naphthoxy)- } \\
{[(R)-4 c]}\end{array}$ & 84 & $\begin{array}{l}{[\alpha]_{\mathrm{D}}^{20}=-49.2^{\circ}} \\
(c=2.4, \mathrm{EtOH})\end{array}$ & $\begin{array}{l}{[\alpha]_{\mathrm{D}}^{20}=+49.6^{\circ},-49.4^{\circ}} \\
(\text { absol. EtOH) })^{15)}\end{array}$ \\
\hline$(R)-3 \mathrm{~d}$ & A & $\begin{array}{l}\text { 2-(2-Naphthoxy)- } \\
{[(R)-4 \mathrm{~d}]}\end{array}$ & 86 & $\begin{array}{l}{[\alpha]_{\mathrm{D}}^{20}=+93.0^{\circ}} \\
(c=2.0, \mathrm{EtOH})\end{array}$ & $\begin{array}{l}{[\alpha]_{\mathrm{D}}^{20}=+93.4^{\circ},-93.3^{\circ}} \\
(\text { absol. EtOH) })^{15)}\end{array}$ \\
\hline
\end{tabular}

Die $O$-Alkylierung mit $(S)-1$ a ist auch auf Di- und Trihydroxybenzole 5 anwendbar, wobei mit ausreichenden Mengen (S)-1a alle OH-Gruppen alkyliert werden. Die erhaltenen Verbindungen $(R)-6$ lassen sich ebenfalls ohne Schwierigkeiten zu den entsprechenden Carbonsäuren $(R)-7$ hydrolysieren. Zur Ermittlung der optischen Ausbeute haben wir 5a-c auch mit racemischem 2-Brompropionsäure-ethylester (1 d) umgesetzt und versucht, die erhaltenen Diastereomerengemische $\left(2 R / S, 2^{\prime} R / S\right)-6 \mathrm{a}-\mathrm{c}$ zu trennen (s.exp. Teil). Dies gelang jedoch eindeutig nur im Falle der Umsetzung mit Brenzcatechin (5a). Daraus ergibt sich für die Umsetzung von $(S)$-1 a mit 5a ein Diastereomerenverhältnis von 12.6:1, das einem 
(R)-Anteil von ca. $3.7 \%$ im eingesetzten Triflat (S)-1 a entspricht. Einen vergleichbaren $(R)$-Anteil haben wir bereits in früheren Untersuchungen ausgehend von käuflicher (S)-Milchsäure festgestellt ${ }^{2 a)}$. In Analogie zu der Umsetzung von (S)-1 a mit $5 \mathbf{a}$ ist auch für die Reaktion mit $5 \mathbf{b}$ und $\mathbf{c}$ unter den angegebenen Bedingungen eine Konfigurationsinversion zu den $(R)$-Verbindungen 6 anzunehmen.

Von den Trihydroxyverbindungen konnten wir am Beispiel von Pyrogallol (5d) zeigen, daß mit der dreifach molaren Menge (S)-1a trotz der zu erwartenden sterischen Hinderung alle drei $\mathrm{OH}-\mathrm{Gruppen}$ zu $6 \mathrm{~d}$ alkyliert werden.

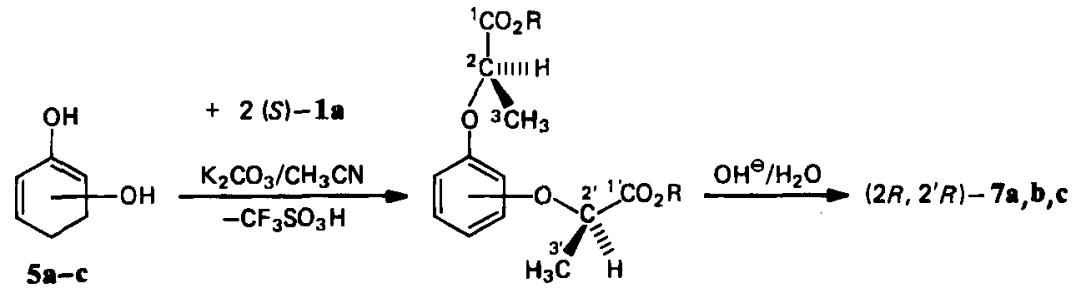

a: ortho b: meta c: para $\quad\left(2 R, 2^{\prime} R\right)-6 \mathrm{a}, \mathrm{b}, \mathrm{c}$

\begin{tabular}{c|cc} 
& $\left(2 R, 2^{\prime} R\right)-6$ & -7 \\
\hline$R$ & $\mathrm{C}_{2} \mathrm{H}_{5}$ & $\mathrm{H}$
\end{tabular}

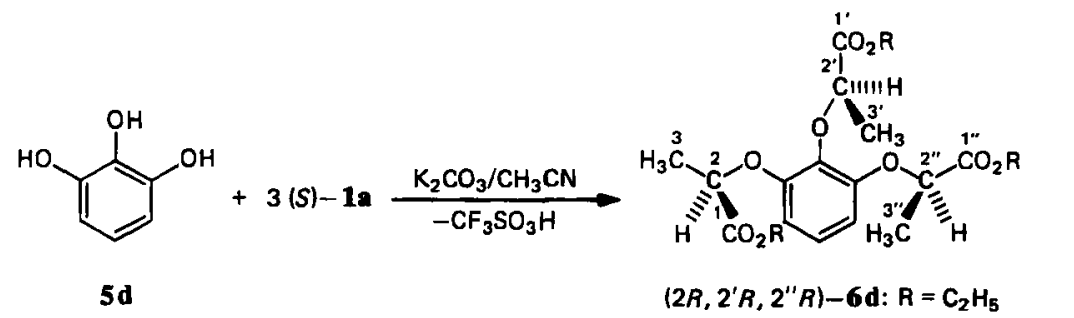

\section{b) Mit Carboxylaten}

Bei der Synthese von Naturstoffen ist häufig ein Austausch der Hydroxygruppe an optisch aktiven Hydroxyverbindungen unter Konfigurationsinversion erforderlich ${ }^{5}$. Hierfür eignen sich besonders gut Carboxylate, mit denen eine geschützte, leicht in die freie Hydroxygruppe überführbare $\mathrm{O}$-Funktion eingeführt werden kann. Vorteilhaft bei dieser Reaktion ist die gegenüber dem Hydroxid-Ion größere Nucleophilie der Carboxylate bei geringerer Basizität, die eine Substitution gegenüber einer Eliminierung begünstigt. Caesiumcarboxylate in Dimethylformamid als Lösungsmittel ergeben erwartungsgemäß die besten Ausbeuten bei solchen Substitutionsreaktionen ${ }^{6}$. Neben Carboxylaten wurden auch geeignete Nitrate mit Sulfonaten optisch aktiver Hydroxy-Verbindungen zu Substitutionsprodukten unter Konfigurationsinversion umgesetzt ${ }^{7,8)}$; hierbei wurden mit den weniger reaktiven Mesylaten bzw. Tosylaten z.T. höhere Racemisierungsanteile beobachtet; z. B. wurde bei den Umsetzungen von Tetrabutylammoniumnitrat mit (S)-2-(Methylsulfonyloxy)propionsäure-ethylester nur ein e.e. von $78 \%$ erhalten ${ }^{7}$, während mit den reaktiveren Trifluormethansulfonaten von Kohlenhydraten keine Racemisierung gefunden wurde ${ }^{8)}$.

Chem. Ber. 119 (1986) 


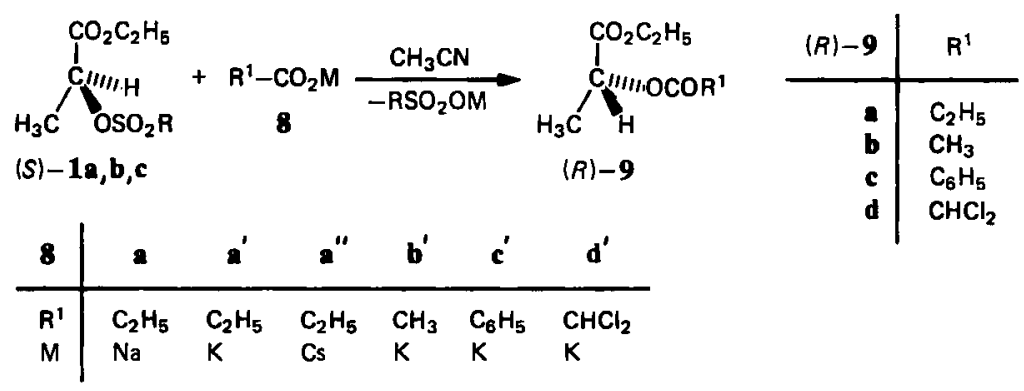

Wir haben nun die (S)-2-(Sulfonyloxy)propionsäureester $(S)-1 \mathbf{a}, \mathbf{b}, \mathbf{c}$ vergleichend mit Natrium- (8a), Kalium- (8a') bzw. Caesiumpropionat (8a') umgesetzt, um zu Aussagen über den Einfluß der Austrittsgruppe in 1 und des Metall-Ions in 8 auf den Verlauf dieser nucleophilen Substitution zu kommen. Die in Tab. 3 aufgeführten Ergebnisse zeigen, daß hierbei in allen Fällen der $(R)-2$-(Propionyloxy)propionsäure-ethylester $[(R)-9 \mathrm{a}]$ in vergleichbarer optischer Reinheit entsteht.

Tab. 3. (R)-(Acyloxy)propionsäure-ethylester (R)-9 aus (S)-2-(Sulfonyloxy)propionsäureethylestern $(S)-1$ und Carboxylaten 8

\begin{tabular}{|c|c|c|c|c|c|c|}
\hline$(S)-1$ & 8 & $\begin{array}{c}\text { Real } \\
\text { Zeit } \\
\text { h }\end{array}$ & $\begin{array}{l}\text { tions- } \\
\text { Temp. } \\
{ }^{\circ} \mathbf{C}\end{array}$ & $\begin{array}{l}\text {-propionsäure- } \\
\text { ethylester }\end{array}$ & Ausb. & $\begin{array}{c}{[\alpha]_{\mathrm{D}}^{20}} \\
(c=5 \\
\left.\mathrm{CHCl}_{3}\right)\end{array}$ \\
\hline$(S)-\mathbf{1} \mathbf{a}$ & $\begin{array}{l}\text { Natrium- } \\
\text { propionat (8a) }\end{array}$ & 20 & $20^{\text {a) }}$ & $\begin{array}{l}\text { (R)-2-(Propionyloxy)- } \\
{[(R)-9 \mathrm{a}]}\end{array}$ & 79 & $+44.4^{\circ}$ \\
\hline$(S)-1 \mathbf{a}$ & $\begin{array}{l}\text { Kalium- } \\
\text { propionat }\left(\mathbf{8 a}^{\prime}\right)\end{array}$ & 2 & $20^{\mathrm{a})}$ & $(R)-9 \mathrm{a}$ & 76 & $+44.4^{\circ}$ \\
\hline$(S)-\mathbf{1} \mathbf{a}$ & $\begin{array}{l}\text { Caesium- } \\
\text { propionat (8a") }\end{array}$ & 2 & $20^{\mathrm{a})}$ & $(R)-9 \mathrm{a}$ & 82 & $+44.8^{\circ}$ \\
\hline$(S)-1 \mathrm{~b}$ & $\mathbf{8 a}$ & 75 & 82 & $\begin{array}{l}\text { unvollständiger } \\
\text { Umsatz }\end{array}$ & & \\
\hline$(S)-1 \mathbf{b}$ & $8 a^{\prime}$ & 24 & 82 & $(R)-9 \mathrm{a}$ & 65 & $+44.0^{\circ}$ \\
\hline$(S)-1 \mathrm{~b}$ & $8 \mathbf{a}^{\prime \prime}$ & 14 & 82 & $(R)-9 \mathbf{a}$ & 76 & $+44.2^{\circ}$ \\
\hline$(S)-1 \mathrm{c}$ & $8 \mathbf{a}$ & 75 & 82 & $\begin{array}{l}\text { unvollständiger } \\
\text { Umsatz }\end{array}$ & & \\
\hline$(S)-1 \mathrm{c}$ & $8 \mathbf{a}^{\prime}$ & 18 & 82 & $(R)-9 \mathbf{a}$ & 73 & $+43.9^{\circ}$ \\
\hline$(S)-1 \mathrm{c}$ & $8 \mathbf{a}^{\prime \prime}$ & 14 & 82 & $(R)-9 \mathrm{a}$ & 71 & $+44.3^{\circ}$ \\
\hline$(S)-1 \mathbf{a}$ & $\begin{array}{l}\text { Kalium- } \\
\text { acetat }\left(\mathbf{8} \mathbf{b}^{\prime}\right)\end{array}$ & 2 & $20^{\text {a) }}$ & $\begin{array}{l}(R)-2 \text {-(Acetoxy)- } \\
{[(R)-9 \mathrm{~b}]}\end{array}$ & 82 & $+49.4^{\circ b}$ \\
\hline$(S)-1 \mathbf{a}$ & $\begin{array}{l}\text { Kalium- } \\
\text { benzoat }\left(\mathbf{8} c^{\prime}\right)\end{array}$ & 7 & $20^{\mathrm{a})}$ & $\begin{array}{l}(R)-2-(\text { Benzoyloxy)- } \\
{[(R)-9 \mathrm{c}]}\end{array}$ & 84 & $-25.6^{\circ \mathrm{c})}$ \\
\hline$(S)-\mathbf{1} \mathbf{a}$ & $\begin{array}{l}\text { Kaliumdichlor- } \\
\text { acetat }\left(\mathbf{8 d} \mathbf{d}^{\prime}\right)\end{array}$ & 3 & $20^{\text {a) }}$ & $\begin{array}{l}(R)-2-(\text { Dichloracetoxy)- } \\
{[(R)-9 \mathrm{~d}]}\end{array}$ & 85 & $+34.1^{\circ}$ \\
\hline
\end{tabular}

a) Zusammengeben der Edukte bei $0^{\circ} \mathrm{C} .-{ }^{\mathrm{b})}[\alpha]_{\mathrm{D}}^{19}$, unverdünnt. $-{ }^{\mathrm{c}}[\alpha]_{\mathrm{D}}^{20}$, unverdünnt. 
Bei den Umsetzungen mit den weniger reaktiven Edukten $(S)-1$ b und $(S)$-1 c sind wiederum härtere Reaktionsbedingungen zur Erzielung befriedigender Ausbeuten erforderlich. Die Abhängigkeit des Reaktionsablaufs vom Metall-Ion war wie erwartet ${ }^{6}$ : Die Umsetzungen von (S)-1 b und (S)-1 c mit Natriumpropionat (8a) waren auch nach 75 h Erhitzen unter Rückfluß $\left(82^{\circ} \mathrm{C}\right)$ nur unvollständig, während mit den reaktiveren Kalium- $\left(\mathbf{8 a}^{\prime}\right)$ und Caesiumpropionaten (8a") bei nur wenig ausgeprägten Reaktivitätsunterschieden zwischen Kalium- und Caesiumsalz bereits nach 14-24 h Erhitzen unter Rückfluß vollständiger Umsatz und vergleichbare Produktausbeuten an $(R)-9$ a erhalten wurden. Die überlegene Reaktivität des Triflats ( $S$ )-1 a zeigte sich bei der Umsetzung mit dem wenig reaktiven Natriumpropionat (8a): bereits nach $20 \mathrm{~h}$ bei $0-20^{\circ} \mathrm{C}$ wird vollständiger Umsatz zu $(R)-9 \mathrm{a}$ mit $79 \%$ Ausb. erreicht.

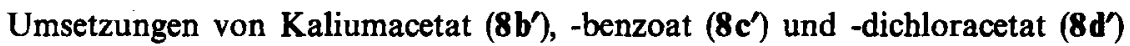
mit $(S)$-1 a führten ebenfalls unter milden Reaktionsbedingungen zu den entsprechenden (R)-(Acyloxy)propionsäure-ethylestern (R)-9b, c, d (Tab. 3). Für die praktische Anwendung kann die Verwendung von Dichloracetaten dann von Vorteil sein, wenn eine selektive hydrolytische Abspaltung des Dichloracetylrestes in Gegenwart anderer Estergruppen erwünscht ist.

Die optische Reinheit der erhaltenen Verbindungen $(R)-9$ wurde durch Vergleich der Drehwerte mit den durch Veresterung aus $(S)$-Milchsäure-ethylester mit den entsprechenden Carbonsäurechloriden erhaltenen Verbindungen ( $S$ )-9 ermittelt. In allen Fällen ist die Übereinstimmung unter Berücksichtigung der Umkehrung des Drehsinnes sehr gut (s. Tab. 3 und exp. Teil). Die Vorteile der erhöhten Reaktivität des Triflats (S)-1 a gegenüber der des Mesylats (S)-1 b und Tosylats (S)-1 c bei Substitutionen an Substraten, die leicht auch Eliminierungen eingehen, konnten wir beispielhaft bei den Umsetzungen des (S)-2-(Trifluormethylsulfonyloxy)bernsteinsäure-diethylesters $[(S)-10 a]$ mit den Kaliumcarboxylaten $\mathbf{8} \mathbf{a}^{\prime}, \mathbf{c}^{\prime}$ zeigen. So verliefen die Umsetzungen von 2-(Methylsulfonyloxy)- (10b) sowie 2-Brombernsteinsäure-diethylester (10d) mit Kaliumpropionat $\left(\mathbf{8} \mathbf{a}^{\prime}\right)$ in Acetonitril bei $20^{\circ} \mathrm{C}$ quantitativ unter Eliminierung zu Fumar- bzw. Maleinsäure-diethylester (11), was auch bei der Umsetzung von 10 b mit Caesiumpropionat (8a") gefunden wurde ${ }^{6}$.

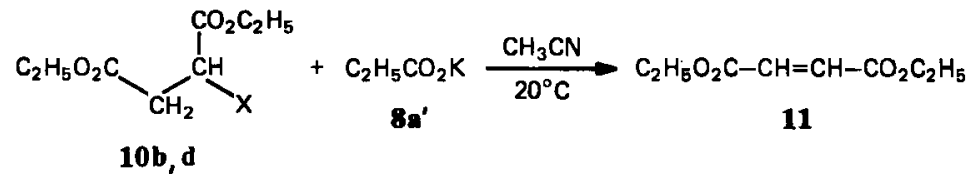

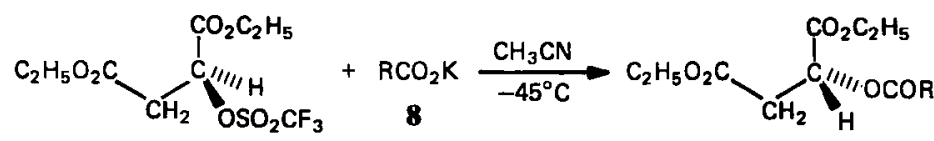

(S)-10a

$(R)-12 a, c$
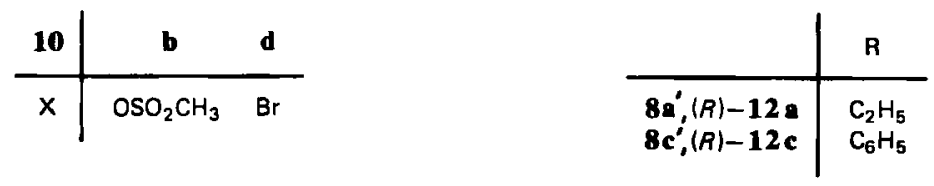
Im Gegensatz dazu gelang bei der Umsetzung des Triflats (S)-10a mit den Kaliumcarboxylaten $8 a^{\prime}$ bzw. $8 c^{\prime}$ in Acetonitril bei $-45^{\circ} \mathrm{C}$ die Substitution zu $(R)$ 2-(Propionyloxy)- $[(R)-12 a]$ bzw. $(R)-2$-(Benzoyloxy)bernsteinsäure-diethylester $[(R)-12 \mathrm{c}]$ mit 52 bzw. $58 \%$ Ausbeute.

Die optische Reinheit der Verbindungen haben wir wiederum durch Vergleich der Drehwerte am Beispiel der auf unabhängigem Wege durch Acylierung von $(S)$-Äpfelsäure-diethylester mit Propionylchlorid erhaltenen Verbindung (S)-12a sichergestellt (s.exp. Teil).

Als Beispiel der racemisierungsfreien Konfigurationsinversion von 2-Hydroxycarbonsäuren mittels der Trifluormethansulfonat-Methode diente die Uberführung von $(S)$ - in $(R)$-Phenylmilchsäure: $(S)$-Phenylmilchsäure $\left[(S)-13,[\alpha]_{\mathrm{D}}^{2 S}=\right.$ $\left.-26.9^{\circ}\right]$ wurde mit Methanol/Salzsäure zum $(S)$-Methylester $(S)-14$ verestert, dieser mit Trifluormethansulfonsäureanhydrid/Pyridin in das Triflat $(S)-15^{2 a)}$ übergeführt, das mit Kaliumacetat (8b') zu $(R)$-2-Acetoxy-3-phenylpropionsäure-methylester $[(R)-16]$ umgesetzt wurde. Dessen alkalische Hydrolyse ergab $(R)$-Phenylmilchsäure $\left[(R)-13,[\alpha]_{\mathbb{D}}^{25}=+26.9^{\circ}\right]$.

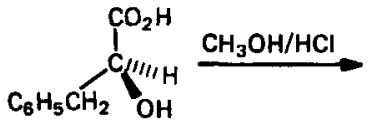

(S) -13<smiles>CCCC[C@@H](O)C(C)=O</smiles>

$(S)-14$<smiles>CO[C@H](Cc1ccccc1)[C@@H](OC(F)(F)F)C(=O)OCC(F)(F)F</smiles>

(S) -15

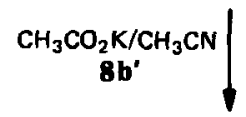

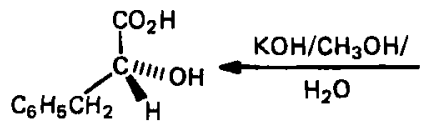

(R)-13<smiles>CC(=O)O[C@H](Cc1ccccc1)C(C)=O</smiles>

(R)-16

\section{c) Mit Schwefelnucleophilen}

Von den in 2-Stellung mit Schwefelfunktionen substituierten Carbonsäuren sind 2-(Arylthio)propionsäuren im Pflanzenschutz ${ }^{3)}$ und 2-(Benzylthio)propionsäuren als Zwischenverbindungen für Pharmaka ${ }^{97}$ bzw. als Bausteine physiologisch interessanter Peptide ${ }^{10)}$ von besonderem Interesse. Für diese Aryl- bzw. Alkylthiosubstituierten Carbonsäuren sind viele Darstellungsmethoden beschrieben, u.a. auch die Substitution von 2-Halogencarbonsäure-Derivaten mit den entsprechen-

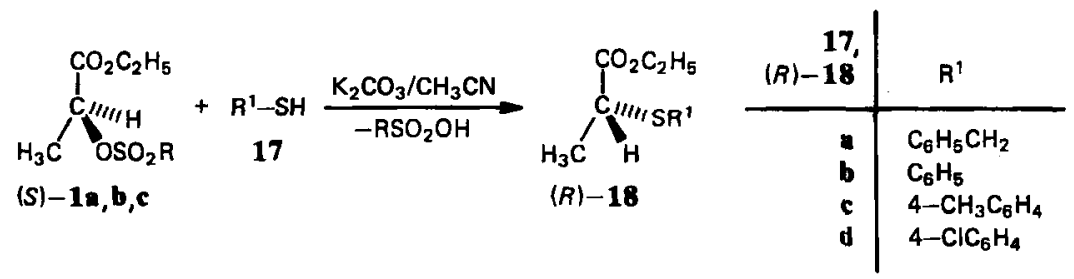


den Schwefelnucleophilen ${ }^{3 b, 11)}$, wobei jedoch meist racemische Halogenverbindungen eingesetzt und in vielen Fällen nur mäßige Ausbeuten erhalten wurden ${ }^{11 c}$. Wir konnten die optisch aktiven Propionsäure-Derivate (S)-1a, b, c sowohl mit Benzolmethanthiol (17a) als auch mit den Thiophenolen 17b-d zu den Substitutionsprodukten ( $R$ )-18 in sehr guten Ausbeuten umsetzen (Tab. 4).

Tab. 4. (R)-2-(Benzylthio)- bzw. (R)-2-(Arylthio)propionsäure-ethylester $(R)-18$ aus $(S)-2-$ (Sulfonyloxy)propionsäure-ethylestern $(S)$-1 und Benzolmethanthiol bzw. Thiophenolen 17

\begin{tabular}{|c|c|c|c|c|c|c|}
\hline$(S)-1$ & 17 & $\begin{array}{c}\text { Real } \\
\text { Zeit } \\
\text { h }\end{array}$ & $\begin{array}{c}\text { ktions- } \\
\text { Temp. } \\
{ }^{\circ} \mathrm{C}\end{array}$ & $\begin{array}{l}\text {-propionsäure- } \\
\text { ethylester }\end{array}$ & $\underset{\%}{\text { Ausb. }}$ & $\begin{array}{c}{[\alpha]_{\mathrm{D}}^{2 \mathrm{~S}}} \\
(c=2, \\
\left.\mathrm{CHCl}]_{3}\right)\end{array}$ \\
\hline$(S)-1 \mathbf{a}$ & $\begin{array}{l}\text { Benzol- } \\
\text { methanthiol } \\
\text { (17a) }\end{array}$ & 20 & $20^{\text {a) }}$ & $\begin{array}{l}(R)-2 \text {-(Benzylthio)- } \\
{[(R)-18 \mathbf{a}]}\end{array}$ & 90 & $+216^{\circ}$ \\
\hline$(S)-1 \mathrm{~b}$ & $17 \mathbf{a}$ & $\begin{array}{l}20 \\
+1.5\end{array}$ & $\begin{array}{l}20^{\mathrm{a})} \\
82\end{array}$ & $(R)-18 \mathbf{a}$ & 83 & $+216^{\circ}$ \\
\hline$(S)-1 \mathrm{c}$ & $17 \mathbf{a}$ & $\begin{array}{l}20 \\
+1.5\end{array}$ & $\begin{array}{l}20^{\text {a) }} \\
82\end{array}$ & $(R)-18 \mathrm{a}$ & 79 & $+210^{\circ}$ \\
\hline$(S)-1 \mathbf{a}$ & $\begin{array}{l}\text { Thiophenol } \\
\text { (17b) }\end{array}$ & 2 & $20^{\text {b) }}$ & $\begin{array}{l}\text { (R)-2-(Phenylthio)- } \\
{[(R)-18 b]}\end{array}$ & 88 & $+132^{\circ}$ \\
\hline$(S)-\mathbf{1 b}$ & $17 b$ & 23 & $20^{\text {a) }}$ & $(R)-18 b$ & 73 & $+133^{\circ}$ \\
\hline$(S)-1 \mathbf{a}$ & $\begin{array}{l}\text { 4-Methyl- } \\
\text { thiophenol } \\
\text { (17c) }\end{array}$ & 2 & $20^{\text {b) }}$ & $\begin{array}{l}(R)-2-(4-M e t h y l p h e n y l t h i o)- \\
{[(R)-18 \mathrm{c}]}\end{array}$ & 88 & $+123^{\circ}$ \\
\hline$(S)-1 \mathrm{~b}$ & $17 \mathrm{c}$ & 25 & $20^{\text {a) }}$ & $(R)-18 c$ & 80 & $+120^{\circ}$ \\
\hline$(S)-1$ a & $\begin{array}{l}\text { 4-Chlor- } \\
\text { thiophenol } \\
\text { (17d) }\end{array}$ & 2 & $20^{\text {b) }}$ & $\begin{array}{l}\text { (R)-2-(4-Chlorphenylthio)- } \\
{[(R)-18 \mathrm{~d}]}\end{array}$ & 87 & $+122^{\circ}$ \\
\hline$(S)-1 \mathrm{~b}$ & 17d & 18 & $20^{\text {a) }}$ & $(R)-18 d$ & 80 & $+122^{\circ}$ \\
\hline
\end{tabular}

a,b) Zusammengeben der Edukte bei a) $0^{\circ} \mathrm{C}$, b) $-45^{\circ} \mathrm{C}$.

ErwartungsgemäB machten sich bei den Reaktionen mit den sehr reaktiven Schwefelnucleophilen die unterschiedlichen Austrittstendenzen des Triflat-Ions einerseits und des Mesylat- bzw. Tosylat-Ions andererseits weniger bemerkbar als bei den Umsetzungen mit den Sauerstoffnucleophilen. So liefen die Substitutionen auch mit $(S)-1$ b, c schon bei Raumtemperatur ab, wobei mit den Thiophenolaten allerdings relativ lange Reaktionszeiten erforderlich wurden (Tab. 4). Die spezifischen Drehwerte der erhaltenen Substitutionsprodukte $(R)-18$ sind weitgehend unabhängig von den eingesetzten Ausgangsverbindungen (S)-1 (Tab. 4), so da $B$ man auf einen einheitlichen sterischen Verlauf der Reaktionen schließen kann.

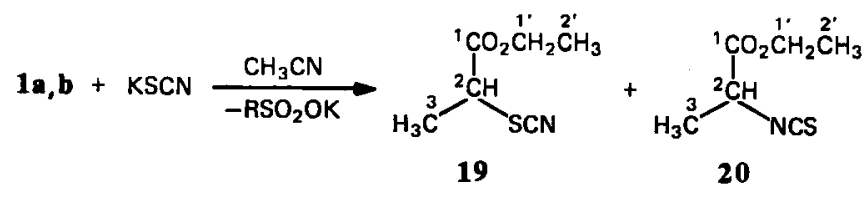

Chem. Ber. 119 (1986) 
Bei den Umsetzungen der Triflate 1a, b mit Kaliumrhodanid als weiterem Schwefelnucleophil reagierte das ambidente Thiocyanat-Ion fast ausschlieBlich am Schwefel, dem Zentrum höherer Nucleophilie, zu 2-(Thiocyanato)propionsäureethylester (19); das dazu isomere Isothiocyanat 20 wurde nur mit $1.8 \%$ im Reaktionsgemisch über gaschromatographische Koinjektion der auf unabhängigem Wege synthetisierten Verbindung ${ }^{12)}$ gefunden. Erwartungsgemäß reagiert auch hier 1 a besser mit Kaliumrhodanid als 1 b (s.exp. Teil). Den sterischen Verlauf dieser Substitutionsreaktion haben wir nicht untersucht, aufgrund der vorstehenden Ergebnisse der Umsetzungen mit den Sauerstoffnucleophilen und Thiolen ist jedoch auch hier eine Substitution unter Konfigurationsinversion anzunehmen.

Wir danken der Deutschen Forschungsgemeinschaft und dem Fonds der Chemischen Industrie für die Förderung und Unterstützung dieser Arbeit.

\section{Experimenteller Teil}

Gaschromatographie: Gaschromatograph Hewlett-Packard, Modell 5700 A und 5710 A mit Flammenionisationsdetektor, Spectra-Physics Minigrator. Trägergas: $30 \mathrm{ml} / \mathrm{min}$ Stickstoff. Glassäulen: $2.3 \mathrm{~m} \times 2 \mathrm{~mm}$ bzw. $2.75 \mathrm{~m} \times 2 \mathrm{~mm}$, Phasen: OV 101/5\% auf Chromosorb W, OV 17/5\% auf Chromosorb W und OV 225/3\% auf Chromosorb W. Carlo Erba Fractovap Modell GI, CE 2150 und EC 4160 mit Flammenionisationsdetektor, Spectra-Physics Minigrator. Trägergas: 0.7 bar Helium, Glaskapillaren $20 \mathrm{~m}$ bzw. $50 \mathrm{~m}$. Phasen: SE 52, SE 54, EM-ON, OV 1701, verschiedene Temperaturprogramme und Heizraten. Präparative Säulenchromatographie: Säulen verschiedener Dimensionen mit Kieselgel S 0.032-0.063 mm (Fa. Riedel-de Haën) und Kieselgel $600.040-0.063 \mathrm{~mm}$ (Fa. Merck). - ${ }^{1}$ H-NMR-Spektren: Geräte Varian T $60(60 \mathrm{MHz})$, Bruker WP 80 (80 MHz), Bruker HX $90(90 \mathrm{MHz})$, Bruker CXP $300(300 \mathrm{MHz}) ; \delta(\mathrm{ppm})$, bezogen auf TMS als int. Standard. $-{ }^{13}$ C-NMR-Spektren: Geräte Bruker HX 90 (22.63 MHz) und CXP 300 (75.47 MHz); $\delta(\mathrm{ppm})$, bezogen auf TMS als int. Standard. - Drehwerte: Perkin-Elmer Polarimeter 241.

(R)-2-(Aryloxy) propionsäure-ethylester (R)-3 (zu Tab. 1): Man läßt bei $0^{\circ} \mathrm{C}$ zu der Lösung des Phenols bzw. Naphthols 2 und Kaliumcarbonat in Acetonitril die Lösung des (S)-2(Sulfonyloxy)propionsäure-ethylesters $(S)$-1 in Acetonitril tropfen, rührt die angegebene Zeit bei $20^{\circ} \mathrm{C}$ bzw. unter Rückfluß bei $82^{\circ} \mathrm{C}$ nach, engt im Rotationsverdampfer ein, gibt zu dem Rückstand Ether und filtriert vom Unlöslichen ab. Die etherische Phase wird mit Wasser gewaschen, über Magnesiumsulfat getrocknet, im Rotationsverdampfer eingeengt und der Rückstand durch Chromatographie über eine Kieselgelsäule oder durch Destillation gereinigt (Tab. 5).

\section{(R)-2-(Aryloxy)propionsäuren (R)-4 (zu Tab. 2)}

Alkalische Hydrolyse von (R)-3a-d: Jeweils $5.0 \mathrm{mmol}(R)-3$ werden bei Raumtemp. mit $10 \mathrm{ml}$ Methanol und $6.25 \mathrm{ml} 2 \mathrm{~N} \mathrm{NaOH} \mathrm{ca} .2 \mathrm{~h}$ gerührt, bis kein $(R)-3 \mathrm{mehr}$ dünnschichtchromatographisch nachweisbar ist. Nach Abdestillieren von Methanol i. Vak. säuert man mit $\mathrm{HCl}$ an und läßt das Gemisch einige $\mathrm{h}$ im Kühlschrank stehen. Man saugt das ausgefallene Produkt ab, wäscht mit Wasser und trocknet i. Hochvak. (Tab. 6).

Saure Hydrolyse von (R)-3a: $1.32 \mathrm{~g}(5.0 \mathrm{mmol})(R)-3 \mathrm{a}$ werden mit $20 \mathrm{ml} \mathrm{konz} . \mathrm{HCl} 20 \mathrm{~h}$ unter Rückfluß erhitzt, anschließend wird die Lösung im Rotationsverdampfer eingeengt und der Rückstand nach Waschen mit Wasser i.Hochvak. getrocknet. Ausb. $0.869 \mathrm{~g}(R)$ 4a, physikalische Daten s. Tab. 6. 
Tab. 5. (R)-2-(Aryloxy)propionsäure-ethylester $(R)-3$

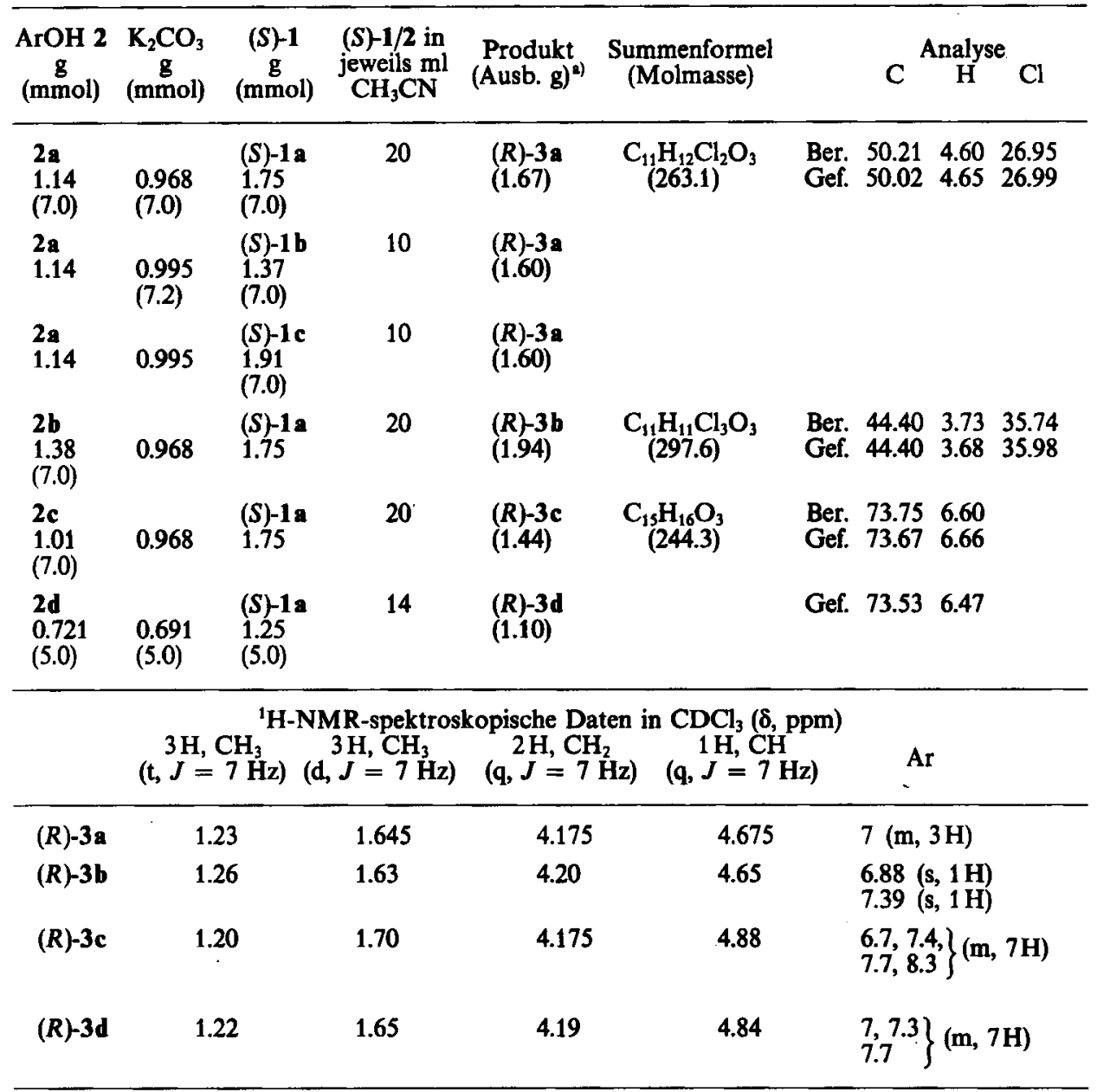

a) Ausb. \% s. Tab. 1.

\section{Umsetzungen von Polyhydroxybenzolen 5}

a) Mit racem. 2-Brompropionsäure-ethylester (1d) zu Diastereomerengemischen der 2,2'[1,2-, 1,3-bzw. 1,4-Phenylenbis(oxy)]/(2R/S, 2'R/S)-bis(propionsäure-ethylester)] 6a-c

1) $2.20 \mathrm{~g}$ (20.0 mmol) Resorcin (5b) wurden mit $6.22 \mathrm{~g}$ (45.0 mmol) Kaliumcarbonat und $8.15 \mathrm{~g}(45.0 \mathrm{mmol})$ racem. $1 \mathrm{~d}$ in $20 \mathrm{ml}$ Acetonitril $25 \mathrm{~h}$ unter Rückfluß erhitzt. Anschließend wurde im Rotationsverdampfer eingeengt, überschüssiger Bromester i. Hochvak. abdestilliert, der Rückstand mit Dichlormethan versetzt und wie bei $(R)-3$ beschrieben aufgearbeitet. Ausb. $4.92 \mathrm{~g}(79 \%)\left(2 R / S, 2^{\prime} R / S\right)-6 \mathrm{~b}$.

2) Jeweils $1.10 \mathrm{~g}(10.0 \mathrm{mmol})$ Brenzcatechin (5a) bzw. Hydrochinon (5c) wurden mit $3.11 \mathrm{~g}$ (22.5 mmol) Kaliumcarbonat und $4.075 \mathrm{~g}(22.5 \mathrm{mmol})$ racem. $1 \mathrm{~d}$ in $10 \mathrm{ml}$ Acetonitril unter

Chem. Ber. 119 (1986) 
Tab. 6. (R)-2-(Aryloxy)propionsäuren (R)-4

\begin{tabular}{|c|c|c|c|c|c|c|c|}
\hline $\begin{array}{c}(R)-3 \mathbf{a} \\
\mathbf{g}\end{array}$ & $\begin{array}{l}\text { Produkt } \\
\text { (Ausb. g) }^{\text {a) }}\end{array}$ & $\underset{\text { (Lit.) }}{\text { Schmp. }}{ }^{\circ} \mathrm{C}$ & $\begin{array}{c}\text { Summenformel } \\
\text { (Molmasse) }\end{array}$ & & $\mathrm{C}^{\mathrm{A}}$ & $\begin{array}{c}\text { Analyse } \\
\mathrm{H}\end{array}$ & $\mathrm{Cl}$ \\
\hline $\begin{array}{l}(R)-3 \mathbf{a} \\
1.31\end{array}$ & $\begin{array}{l}(R)-4 \mathrm{a} \\
(0.994)\end{array}$ & $\begin{array}{l}122-123 \\
(122-123,(+)-\text { bzw. } \\
(-) \text {-Form, Racemat } \\
(117-118)^{13)}\end{array}$ & $\begin{array}{l}\mathrm{C}_{9} \mathrm{H}_{8} \mathrm{Cl}_{2} \mathrm{O}_{3} \\
(\mathbf{1 3 5 . 1 )}\end{array}$ & $\begin{array}{l}\text { Ber. } \\
\text { Gef. }\end{array}$ & $\begin{array}{l}45.99 \\
45.79\end{array}$ & $\begin{array}{l}3.43 \\
3.55\end{array}$ & $\begin{array}{l}30.16 \\
30.04\end{array}$ \\
\hline $\begin{array}{l}(\boldsymbol{R})-\mathbf{3 b} \\
1.49\end{array}$ & $\begin{array}{l}(R)-4 \mathrm{~b} \\
(1.09)\end{array}$ & $\begin{array}{l}145 \\
(144-145)^{14)}\end{array}$ & $\begin{array}{c}\mathrm{C}_{9} \mathrm{H}_{7} \mathrm{Cl}_{3} \mathrm{O}_{3} \\
(269.5)\end{array}$ & $\begin{array}{l}\text { Ber. } \\
\text { Gef. }\end{array}$ & $\begin{array}{l}40.11 \\
40.31\end{array}$ & $\begin{array}{l}2.61 \\
2.61\end{array}$ & $\begin{array}{l}39.46 \\
39.31\end{array}$ \\
\hline $\begin{array}{l}(R)-3 \mathrm{c} \\
1.22\end{array}$ & $\begin{array}{l}(R)-4 c \\
(0.908)\end{array}$ & 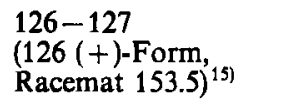 & $\begin{array}{r}\mathrm{C}_{13} \mathrm{H}_{12} \mathrm{O}_{3} \\
(216.2)\end{array}$ & $\begin{array}{l}\text { Ber. } \\
\text { Gef. }\end{array}$ & $\begin{array}{l}72.21 \\
71.99\end{array}$ & $\begin{array}{l}5.59 \\
5.79\end{array}$ & \\
\hline $\begin{array}{l}(R)-3 \mathrm{~d} \\
1.22\end{array}$ & $\begin{array}{l}(R)-4 d \\
(0.929)\end{array}$ & $\begin{array}{l}118 \\
(119(+) \text {-Form, } \\
\text { Racemat } 108.5,114)^{15)}\end{array}$ & $\begin{array}{r}\mathrm{C}_{13} \mathrm{H}_{12} \mathrm{O}_{3} \\
(216.2)\end{array}$ & $\begin{array}{l}\text { Ber. } \\
\text { Gef. }\end{array}$ & $\begin{array}{l}72.21 \\
72.08\end{array}$ & $\begin{array}{l}5.59 \\
5.59\end{array}$ & \\
\hline
\end{tabular}

H-NMR-spektroskopische Daten in $\mathrm{CDCl}_{3}(\delta, \mathrm{ppm})$ $3 \mathrm{H}, \mathrm{CH}_{3} \quad 1 \mathrm{H}, \mathrm{CH}$ $(\mathrm{d}, J=7 \mathrm{~Hz})(\mathrm{q}, J=7 \mathrm{~Hz})$

$1 \mathrm{H}, \mathrm{CO}_{2} \mathrm{H}$

(s)

\begin{tabular}{llllr}
\hline$(R)-4 \mathbf{a}$ & 1.73 & 4.77 & $7(\mathrm{~m}, 3 \mathrm{H})$ & 9.8 \\
$(R)-\mathbf{4 b}$ & 1.70 & 4.83 & $7.05(\mathrm{~s}, 1 \mathrm{H}), 7.5(\mathrm{~s}, 1 \mathrm{H})$ & 9.6 \\
$(R)-4 \mathrm{c}$ & 1.65 & 4.80 & $6.6,7.4,7.7,8.2(\mathrm{~m}, 7 \mathrm{H})$ & 10.7 \\
$(R)-4 \mathrm{~d}$ & 1.67 & 4.86 & $7,7.3,7.7(\mathrm{~m}, 7 \mathrm{H})$ & 11.2
\end{tabular}

a) Ausb. \% s. Tab. 2.

Rückfluß erhitzt (5a 25 h, 5c 14 h) und wie unter 1) beschrieben aufgearbeitet. Ausb. Umsetzung mit 5a: $1.80 \mathrm{~g}(58 \%)\left(2 R / S, 2^{\prime} R / S\right) 6 \mathrm{a}$, mit $5 \mathrm{c}: 2.54 \mathrm{~g}(82 \%)\left(2 R / S, 2^{\prime} R / S\right)-6 \mathrm{c}$.

Spektroskopische Daten von $6(\delta, \mathrm{ppm})$

${ }^{1} \mathrm{H}-\mathrm{NMR} \quad 6 \mathrm{H}, 2 \mathrm{CH}_{3} \quad 6 \mathrm{H}, 2 \mathrm{CH}_{3} \quad 4 \mathrm{H}, 2 \mathrm{CH}_{2} \quad 2 \mathrm{H}, 2 \mathrm{CH}$

$\left(\mathrm{CDCl}_{3}\right) \quad(\mathrm{t}, J=7 \mathrm{~Hz})(\mathrm{d}, J=7 \mathrm{~Hz})(\mathrm{q}, J=7 \mathrm{~Hz})(\mathrm{q}, J=7 \mathrm{~Hz})$

Ph

\begin{tabular}{|c|c|c|c|c|c|}
\hline$\left.\left(2 R, 2^{\prime} R\right)-6 \mathrm{a}^{\mathrm{a}}\right)$ & 1.24 & 1.65 & $4.19(d q)$ & 4.82 & $6.93(\mathrm{~s}, 4 \mathrm{H})$ \\
\hline $\begin{array}{l}\left(2 R / S, 2^{\prime} R / S\right)-6 \mathrm{a} \\
\quad(1: 1)\end{array}$ & $\begin{array}{l}1.24 \\
1.26\end{array}$ & $\begin{array}{l}1.60 \\
1.65\end{array}$ & $4.2(\mathrm{~m})$ & $\begin{array}{l}4.82 \\
4.824\end{array}$ & $6.92(\mathrm{~s}, 4 \mathrm{H})$ \\
\hline$\left(2 R, 2^{\prime} R\right)-6 \mathrm{~b}$ & 1.25 & 1.59 & 4.2 & 4.70 & $\begin{array}{l}6.5(\mathrm{~m}, 3 \mathrm{H}) \\
7.16(\mathrm{t}, 1 \mathrm{H}, o-\mathrm{H})\end{array}$ \\
\hline $\begin{array}{l}\left(2 R / S, 2^{\prime} R / S\right)-6 b \\
(1: 1)\end{array}$ & 1.25 & 1.59 & 4.2 & $\begin{array}{l}4.705 \\
4.712\end{array}$ & $\begin{array}{l}6.5(\mathrm{~m}, 3 \mathrm{H}) \\
7.16(\mathrm{t}, 1 \mathrm{H}, \mathrm{o}-\mathrm{H})\end{array}$ \\
\hline$\left(2 R, 2^{\prime} R\right)-6 \mathrm{c}$ & 1.24 & 1.58 & 4.2 & 4.64 & $6.8(\mathrm{~s}, 4 \mathrm{H})$ \\
\hline $\begin{array}{l}\left(2 R / S, 2^{\prime} R / S\right)-6 \mathrm{c} \\
\quad(1: 1)\end{array}$ & 1.24 & 1.58 & 4.2 & 4.64 & $6.8(\mathrm{~s}, 4 \mathrm{H})$ \\
\hline
\end{tabular}

Chem. Ber. 119 (1986) 


\begin{tabular}{|c|c|c|c|c|c|c|}
\hline $\begin{array}{l}{ }^{13} \mathrm{C}-\mathrm{NMR} \\
\left(\mathrm{CDCl}_{3}\right)\end{array}$ & $\mathrm{C}=\mathrm{O}$ & $\mathrm{Ph}$ & $\mathrm{CH}$ & $\mathrm{CH}_{2}$ & $\mathrm{CH}_{3} \mathrm{CH}$ & $\mathrm{CH}_{3} \mathrm{CH}_{2}$ \\
\hline$\left(2 R, 2^{\prime} R\right)-6 \mathbf{a}$ & 172.29 & $\begin{array}{l}148.62(\mathrm{C}-1,-2) \\
122.89(\mathrm{C}-4,-5) \\
118.03(\mathrm{C}-3,-6)\end{array}$ & 74.65 & 61.03 & 18.63 & 14.14 \\
\hline$\left(2 R / S, 2^{\prime} R / S\right)-6 a$ & $\begin{array}{l}172.29 \\
172.17\end{array}$ & $\begin{array}{l}148.65(\mathrm{C}-1) \\
148.38(\mathrm{C}-2) \\
122.90(\mathrm{C}-4,-5) \\
118.27(\mathrm{C}-3) \\
118.07(\mathrm{C}-6)\end{array}$ & $\begin{array}{l}74.68 \\
74.66\end{array}$ & 61.05 & $\begin{array}{l}18.64 \\
18.47\end{array}$ & 14.16 \\
\hline$\left(2 R, 2^{\prime} R\right)-6 b$ & 172.05 & $\begin{array}{l}158.94(C-1,-3) \\
130.01(C-5) \\
108.34(C-4,-6) \\
103.41(C-2)\end{array}$ & 72.88 & 61.26 & 18.49 & 14.12 \\
\hline$\left(2 R / S, 2^{\prime} R / S\right)-6 \mathrm{~b}$ & 172.04 & $\begin{array}{l}158.91(\mathrm{C}-1,-3) \\
129.99(\mathrm{C}-5) \\
108.28(\mathrm{C}-4) \\
108.21(\mathrm{C}-6) \\
103.40(\mathrm{C}-2) \\
103.03(\mathrm{C})\end{array}$ & 72.73 & 61.23 & 18.50 & 14.11 \\
\hline$\left(2 R, 2^{\prime} R\right)-6 \mathrm{c}$ & 172.31 & $\begin{array}{c}152.26(C-1,-4) \\
116.57(C-2,-3) \\
-5,-6)\end{array}$ & 73.69 & 61.18 & 18.59 & 14.14 \\
\hline$\left(2 R / S, 2^{\prime} R / S\right)-6 \mathrm{c}$ & 172.30 & $\begin{array}{c}152.57(C-1,-4) \\
116.57(C-2,-3, \\
-5,-6)\end{array}$ & $73.70^{\text {b) }}$ & $61.16^{\text {b) }}$ & 18.58 & 14.13 \\
\hline $\begin{array}{c}\mathrm{C}_{16} \mathrm{H}_{22} \mathrm{O}_{6} \\
(310.3)\end{array}$ & & \multicolumn{2}{|c|}{ Ber. C 61.92 H 7.15} & \multicolumn{3}{|c|}{$\begin{array}{c}{[\alpha]_{\mathrm{D}}^{20}} \\
\left(c=3, \stackrel{\mathrm{C}}{\mathrm{C}} \mathrm{HCl}_{3}\right)\end{array}$} \\
\hline $\begin{array}{l}\left(2 R, 2^{\prime} R\right)-6 \mathrm{a} \\
\left(2 R, 2^{\prime} R\right)-6 \mathrm{~b} \\
\left(2 R, 2^{\prime} R\right)-6 \mathrm{c}\end{array}$ & & \multicolumn{2}{|c|}{$\begin{array}{l}\text { Gef. C } 62.05 \text { H } 7.26 \\
\text { Gef. C } 61.80 \text { H } 7.30 \\
\text { Gef. C } 61.93 \text { H } 7.26\end{array}$} & \multicolumn{3}{|c|}{$\begin{array}{l}+81.3^{\circ} \\
+39.7^{\circ} \\
+54.0^{\circ}\end{array}$} \\
\hline
\end{tabular}

a) Diastereomerenanteil nach ${ }^{1} \mathrm{H}-\mathrm{NMR}$ ca. 5-10\%. - ${ }^{\text {b) }}$ Trennung ,angedeutet“".

b) Mit (S)-2-(Trifluormethylsulfonyloxy) propionsäure-ethylester [(S)-1 a]: Man tropft zu jeweils $1.10 \mathrm{~g}(10.0 \mathrm{mmol}) 5 \mathrm{a}, \mathrm{b}$ bzw. $\mathrm{c}$ und $3.32 \mathrm{~g}(24.0 \mathrm{mmol})$ Kaliumcarbonat in $40 \mathrm{ml}$ Acetonitril bei Raumtemp. innerhalb $10 \mathrm{~min}$ die Lösung von $5.0 \mathrm{~g}(20.0 \mathrm{mmol})(S)-1 \mathrm{a}$ in $40 \mathrm{ml}$ Acetonitril, rührt ca. $20 \mathrm{~h}$ bei Raumtemp., engt im Rotationsverdampfer ein, gibt zum Rückstand Dichlormethan und arbeitet wie bei $(R)-3$ beschrieben auf. Ausb. $2.80 \mathrm{~g}(90 \%)$ $\left(2 R, 2^{\prime} R\right)-6 \mathrm{a}, 2.84 \mathrm{~g}(92 \%)\left(2 R, 2^{\prime} R\right)-6 \mathrm{~b}$ bzw. $2.80 \mathrm{~g}(90 \%)\left(2 R, 2^{\prime} R\right)-6 \mathrm{c}$. Gaschromatographisch wurde aus den Flächenintegralen bei $\left(2 R, 2^{\prime} R\right)-6 \mathrm{a}$ ein Diastereomerenverhältnis von 12.6:1 bestimmt, entsprechend einem Anteil von 7.6\% 2,2'-[1,2-Phenylenbis(oxy)][(2R/S, $2^{\prime} R / S$ )-bis(propionsäure-ethylester)].

2,2'-[1,2-, 1,3- und 1,4-Phenylenbis (oxy) $]\left[\left(2 R, 2^{\prime} R\right)-\right.$ bis (propionsäure) $](7 \mathbf{a}, \mathbf{b}, \mathbf{c})$ : Jeweils $2.56 \mathrm{~g}(8.25 \mathrm{mmol})\left(2 R, 2^{\prime} R\right)-6 \mathrm{a},-6 \mathrm{~b} \mathrm{bzw},-6 \mathrm{c}$ werden bei Raumtemp. mit $10 \mathrm{ml}$ Methanol und $10 \mathrm{ml} 2 \mathrm{~N} \mathrm{NaOH} \mathrm{ca.} 1.5 \mathrm{~h}$ gerührt, bis kein Ester mehr dünnschichtchromatographisch nachweisbar ist. Nach Abdestillieren des Methanols säuert man mit $\mathrm{HCl}$ an, extrahiert mit 
Dichlormethan, trocknet den Extrakt über Natriumsulfat, entfernt das Lösungsmittel im Rotationsverdampfer, pulverisiert den Rückstand, wäscht mit $n$-Pentan und trocknet i. Hochvak. Ausb. $1.6 \mathrm{~g}(76 \%)\left(2 R, 2^{\prime} R\right)-7 \mathrm{a}, 1.4 \mathrm{~g} \mathrm{(67 \% )}\left(2 R, 2^{\prime} R\right)-7 \mathrm{~b}$ bzw. $1.02 \mathrm{~g} \mathrm{(49 \% )}(2 R$, $\left.2^{\prime} R\right)-7 \mathrm{c}$.

Spektroskopische Daten von 7 ( $\delta, \mathrm{ppm})$

\begin{tabular}{|c|c|c|c|c|}
\hline $\begin{array}{c}{ }^{1} \mathrm{H}-\mathrm{NMR} \\
\left(\left[\mathrm{D}_{6}\right] \text { Aceton) }\right.\end{array}$ & $\begin{array}{c}3 \mathrm{H}, \mathrm{CH}_{3} \\
(\mathrm{~d}, J=7 \mathrm{~Hz})\end{array}$ & $\begin{array}{c}1 \mathrm{H}, \mathrm{CH} \\
(\mathrm{q}, J=7 \mathrm{~Hz})\end{array}$ & $\mathbf{P h}$ & $1 \mathrm{H}, \mathrm{OH}$ \\
\hline$\left(2 R, 2^{\prime} R\right)-7 \mathbf{a}$ & 1.61 & 4.90 & \multirow{2}{*}{$\begin{array}{l}7.0(\mathrm{~m}, 4 \mathrm{H}) \\
6.5(\mathrm{~m}, 3 \mathrm{H}) \\
7.16(\mathrm{t}, 1 \mathrm{H}, o-\mathrm{H})\end{array}$} & 9.0 \\
\hline$\left(2 R, 2^{\prime} R\right)-7 \mathrm{~b}$ & 1.57 & 4.81 & & 9.9 \\
\hline$\left(2 R, 2^{\prime} R\right)-7 \mathrm{c}$ & 1.55 & 4.75 & $6.86(\mathrm{~s}, 4 \mathrm{H})$ & 9.3 \\
\hline $\begin{array}{c}{ }^{13} \mathrm{C}-\mathrm{NMR} \\
\left.{ }_{(\mathrm{CDCl}}\right)\end{array}$ & $\mathrm{C}=\mathrm{O}$ & $\mathrm{Ph}$ & $\mathrm{CH}$ & $\mathrm{CH}_{3}$ \\
\hline$\left(2 R, 2^{\prime} R\right)-7 \mathrm{a}$ & 173.42 & $\begin{array}{l}129.58(C-1,-2) \\
123.54(C-4,-5) \\
118.61(C-3,-6)\end{array}$ & 75.09 & 18.86 \\
\hline$\left(2 R, 2^{\prime} R\right)-7 b$ & 173.14 & $\begin{array}{l}160.07(C-1,-3) \\
130.75(C-5) \\
108.73(C-4,-6) \\
103.76(C-2)\end{array}$ & 73.00 & 18.74 \\
\hline$\left(2 R, 2^{\prime} R\right)-7 \mathrm{c}$ & 173.39 & $\begin{array}{l}153.44(\mathrm{C}-1,-4) \\
117.09(\mathrm{C}-2,-3,\end{array}$ & $-6)$ & 18.85 \\
\hline
\end{tabular}

\begin{tabular}{cccc}
$\begin{array}{c}\mathrm{C}_{12} \mathrm{H}_{14} \mathrm{O}_{6} \\
(254.2)\end{array}$ & Ber. C $56.69 \mathrm{H} 5.55$ & $\begin{array}{c}{[\alpha]_{\mathrm{D}}^{20}} \\
(c=1.6, \text { Aceton })\end{array}$ & Schmp. ${ }^{\circ} \mathrm{C}$ \\
\hline$\left(2 R, 2^{\prime} R\right)-7 \mathrm{a}$ & Gef. C 56.44 H 5.76 & $+70.8^{\circ}$ & $148-150$ \\
$\left(2 R, 2^{\prime} R\right)-7 \mathrm{~b}$ & Gef. C $56.24 \mathrm{H} 5.61$ & $+54.9^{\circ}$ & $132-140$ \\
$\left(2 R, 2^{\prime} R\right)-7 \mathrm{c}$ & Gef. C $56.48 \mathrm{H} 5.74$ & $+89.0^{\circ}$ & $\begin{array}{l}\text { ca. 160 (ab ca. } 110 \\
\end{array}$ \\
& & & Erweichung) \\
\hline
\end{tabular}

$2,2^{\prime}, 2^{\prime \prime}-[1,2,3-B e n z o l t r i y l t r i s(o x y)] /\left(2 R, 2^{\prime} R, 2^{\prime \prime} R\right)-$ tris (propionsäure-ethylester) $]\left[\left(2 R, 2^{\prime} R\right.\right.$, $\left.2^{\prime \prime} R\right)-6 \mathrm{~d}$ ]: Bei Raumtemp. pipettiert man zu $0.25 \mathrm{~g}$ (2.0 mmol) Pyrogallol (5d) und $0.97 \mathrm{~g}$ (7.0 mmol) Kaliumcarbonat in $10 \mathrm{ml}$ Acetonitril $1.50 \mathrm{~g}(6.0 \mathrm{mmol})(S)-1 \mathrm{a}$, rührt $2 \mathrm{~d}$ bei Raumtemp., engt im Rotationsverdampfer ein, gibt zu dem Rückstand Dichlormethan und arbeitet wie bei $(R)-3$ angegeben auf. Ausb. $0.62 \mathrm{~g}(73 \%)$. $-{ }^{1} \mathrm{H}-\mathrm{NMR}\left(\mathrm{CDCl}_{3}\right): \delta=1.23(\mathrm{t}$, $\left.J=7 \mathrm{~Hz}, 6 \mathrm{H}, 2 \mathrm{CH}_{3}\right), 1.25\left(\mathrm{t}, J=7 \mathrm{~Hz}, 3 \mathrm{H}, \mathrm{CH}_{3}\right), 1.59\left(\mathrm{~d}, J=7 \mathrm{~Hz}, 3 \mathrm{H}, \mathrm{CH}_{3}\right), 1.60$ (d, $J=7 \mathrm{~Hz}, 6 \mathrm{H}, 2 \mathrm{CH}_{3}$ ), 4.20 und 4.21 (jeweils q, $\left.6 \mathrm{H}, 3 \mathrm{CH}_{2}\right), 4.78(\mathrm{q}, J=7 \mathrm{~Hz}, 3 \mathrm{H}, 3 \mathrm{CH}$ ), 6.50 (d, 2H, Ph), $6.86(\mathrm{t}, 1 \mathrm{H}, \mathrm{Ph}) .-{ }^{13} \mathrm{C}-\mathrm{NMR}\left(\mathrm{CDCl}_{3}\right): \delta=172.06,172.00(3 \mathrm{C}=\mathrm{O}), 151.67$ (Ph, C-1, -3), 138.44 (Ph, C-2), 123.49 (Ph, C-5), $109.80(\mathrm{Ph}, \mathrm{C}-4,-6), 73.99$ (3 CH), 61.10, $60.70\left(3 \mathrm{CH}_{2}\right), 18.49,18.41\left(3 \mathrm{CH}_{3} \mathrm{CH}\right), 14.17,14.10\left(3 \mathrm{CH}_{3} \mathrm{CH}_{2}\right)$.

$\mathrm{C}_{21} \mathrm{H}_{30} \mathrm{O}$, (426.5) Ber. C 59.14 H 7.09 Gef. C 59.02 H 7.19

(R)-2-(Acyloxy)propionsäure-ethylester (R)-9 (zu Tab. 3): Man läßt zu der Lösung des Carboxylats 8 in Acetonitril die Lösung von $(S)-1$ in Acetonitril tropfen und setzt wie bei 
Tab. 7. (R)-2-(Acyloxy)propionsäure-ethylester $(R)-9$

\begin{tabular}{|c|c|c|c|c|c|c|c|c|c|}
\hline $\begin{array}{c}\mathbf{8} \\
\mathbf{g} \\
\text { (mmol) }\end{array}$ & $\begin{array}{c}(S)-1 \\
\mathbf{g} \\
(\mathrm{mmol})\end{array}$ & $\begin{array}{l}8 /(S)-1 \text { in } \\
\text { jeweils ml } \\
\mathrm{CH}_{3} \mathrm{CN}\end{array}$ & $\begin{array}{c}\text { Produkt } \\
\text { (Ausb. g) }^{\text {A) }}\end{array}$ & $\begin{array}{l}\text { Sdp. } \\
{ }^{\circ} \text { C/Torr } \\
\text { (Lit.) }\end{array}$ & $\begin{array}{l}\text { Summenformel } \\
\text { (Molmasse) }\end{array}$ & & $\mathrm{C}^{\mathrm{A}}$ & $\underset{\mathrm{H}}{\text { Analyse }}$ & $\mathrm{Cl}$ \\
\hline $\begin{array}{l}\mathbf{8 a} \\
1.15 \\
(12.0)\end{array}$ & $\begin{array}{l}(S)-1 \mathbf{a} \\
2.5 \\
(10.0)\end{array}$ & $20 / 10$ & $\begin{array}{l}(R)-9 \mathbf{a} \\
(1.38)\end{array}$ & $75 / 10$ & $\begin{array}{r}\mathrm{C}_{8} \mathrm{H}_{14} \mathrm{O}_{4} \\
(174.2)\end{array}$ & $\begin{array}{l}\text { Ber. } \\
\text { Gef. }\end{array}$ & $\begin{array}{l}55.16 \\
55.22\end{array}$ & $\begin{array}{l}8.10 \\
8.38\end{array}$ & \\
\hline $\begin{array}{l}8 \mathbf{a}^{\prime} \\
3.37 \\
(30.0)\end{array}$ & $\begin{array}{l}(S)-1 \mathrm{a} \\
6.25 \\
(25.0)\end{array}$ & $50 / 30$ & $\begin{array}{l}(R)-9 \mathrm{a} \\
(3.31)\end{array}$ & & & & & & \\
\hline $\begin{array}{l}8 \mathbf{a}^{\prime \prime} \\
2.47 \\
(12.0)\end{array}$ & $\begin{array}{l}(S)-1 \mathbf{a} \\
2.5\end{array}$ & $20 / 12$ & $\begin{array}{l}(R)-9 \mathrm{a} \\
(1.43)\end{array}$ & & & & & & \\
\hline $\begin{array}{l}8 \mathbf{a} \\
1.15\end{array}$ & $\begin{array}{l}(S)-1 \mathrm{~b} \\
1.96 \\
(10.0)\end{array}$ & $16 / 16$ & $\begin{array}{l}\text { unvoll- } \\
\text { ständiger } \\
\text { Umsatz }\end{array}$ & & & & & & \\
\hline $\begin{array}{l}\mathbf{8} \mathbf{a}^{\prime} \\
3.37\end{array}$ & $\begin{array}{l}(S)-1 \text { b } \\
4.91 \\
(25.0)\end{array}$ & $80 / 80$ & $\begin{array}{l}(R)-9 \mathrm{a} \\
(2.83)\end{array}$ & & & & & & \\
\hline $\begin{array}{l}8 \mathbf{a}^{\prime \prime} \\
2.47\end{array}$ & $\begin{array}{l}(S)-1 \text { b } \\
1.96\end{array}$ & $20 / 12$ & $\begin{array}{l}(R)-9 \mathbf{a} \\
(1.33)\end{array}$ & & & & & & \\
\hline $\begin{array}{l}8 \mathrm{a} \\
1.15\end{array}$ & $\begin{array}{l}(S)-1 \mathrm{c} \\
2.72 \\
(10.0)\end{array}$ & $16 / 16$ & $\begin{array}{l}\text { unvoll- } \\
\text { ständiger } \\
\text { Umsatz }\end{array}$ & & & & & & \\
\hline $\begin{array}{l}\mathbf{8} \mathbf{a}^{\prime} \\
3.37\end{array}$ & $\begin{array}{l}(S)-1 \mathrm{c} \\
6.81 \\
(25.0)\end{array}$ & $80 / 80$ & $\begin{array}{l}(R)-9 \mathrm{a} \\
(3.18)\end{array}$ & & & & & & \\
\hline $\begin{array}{l}8 a^{\prime \prime} \\
2.47\end{array}$ & $\begin{array}{l}(S)-1 \mathrm{c} \\
2.72\end{array}$ & $20 / 12$ & $\begin{array}{l}(R)-9 \mathrm{a} \\
(1.24)\end{array}$ & & & & & & \\
\hline $\begin{array}{l}\mathbf{8 b}^{\prime} \\
2.94 \\
(30.0)\end{array}$ & $\begin{array}{l}(S)-1 \mathrm{a} \\
6.25\end{array}$ & $50 / 30$ & $\begin{array}{l}(R)-9 \mathrm{~b} \\
(3.30)\end{array}$ & $\begin{array}{l}65 / 12 \\
(73-74 / 11, \\
\text { "d“-Form) }\end{array}$ & $\begin{array}{r}\mathrm{C}_{7} \mathrm{H}_{12} \mathrm{O}_{4} \\
(160.2)\end{array}$ & $\begin{array}{l}\text { Ber. } \\
\text { Gef. }\end{array}$ & $\begin{array}{l}52.49 \\
52.48\end{array}$ & $\begin{array}{l}7.55 \\
7.59\end{array}$ & \\
\hline $\begin{array}{l}8 c^{\prime} \\
4.81 \\
(30.0)\end{array}$ & $\begin{array}{l}(S)-1 \mathrm{a} \\
6.25\end{array}$ & $50 / 30$ & $\begin{array}{l}(R)-9 \mathrm{c} \\
(4.67)\end{array}$ & $\begin{array}{l}80 / 10^{-2} \\
(153 / 12, \\
\text { „d"-Form) }{ }^{17)}\end{array}$ & $\begin{array}{r}\mathrm{C}_{12} \mathrm{H}_{14} \mathrm{O}_{4} \\
(222.2)\end{array}$ & $\begin{array}{l}\text { Ber. } \\
\text { Gef. }\end{array}$ & $\begin{array}{l}64.85 \\
64.62\end{array}$ & $\begin{array}{l}6.35 \\
6.21\end{array}$ & \\
\hline $\begin{array}{l}8 d^{\prime} \\
1.0 \\
(6.0)\end{array}$ & $\begin{array}{l}(S)-1 \mathrm{a} \\
1.25 \\
(5.0)\end{array}$ & $15 / 10$ & $\begin{array}{l}(R)-9 \mathrm{~d} \\
(0.97)\end{array}$ & $\begin{array}{l}\text { farbl. } \\
\text { Ôl }\end{array}$ & $\underset{(229.1)}{\mathrm{C}_{7} \mathrm{H}_{10} \mathrm{Cl}_{2} \mathrm{O}_{4}}$ & $\begin{array}{l}\text { Ber. } \\
\text { Gef. }\end{array}$ & $\begin{array}{l}36.71 \\
36.68\end{array}$ & $\begin{array}{l}4.40 \\
4.49\end{array}$ & $\begin{array}{l}30.96 \\
30.94\end{array}$ \\
\hline
\end{tabular}

${ }^{1} \mathrm{H}-\mathrm{NMR}$-spektroskopische Daten in $\mathrm{CDCl}_{3}(\delta, \mathrm{ppm})$

\begin{tabular}{|c|c|c|c|c|}
\hline $\begin{array}{c}3 \mathrm{H} \\
\mathrm{CH}_{3} \mathrm{CH}_{2} \\
(\mathrm{t}, \mathrm{J}= \\
7 \mathrm{~Hz})\end{array}$ & $\begin{array}{c}3 \mathrm{H}, \\
\mathrm{CH} H_{3} \mathrm{CH} \\
(\mathrm{d}, J= \\
7 \mathrm{~Hz})\end{array}$ & $\begin{array}{c}3 \mathrm{H}, \\
\mathrm{CH}_{3} \mathrm{CO} \\
(\mathrm{s})\end{array}$ & $\begin{array}{c}2 \mathrm{H}, \\
\mathrm{CH}_{3} \mathrm{CH} H_{2} \\
(\mathrm{q}, \mathrm{J}= \\
7 \mathrm{~Hz})\end{array}$ & $\begin{array}{c}1 \mathrm{H}, \mathrm{CH} \\
(\mathrm{q}, J= \\
7 \mathrm{~Hz})\end{array}$ \\
\hline
\end{tabular}

\begin{tabular}{|c|c|c|c|c|c|c|}
\hline$(R)-9 \mathrm{a}$ & $\begin{array}{l}1.17 \\
1.28\end{array}$ & 1.47 & & $\begin{array}{l}2.41(\mathrm{dq}) \\
4.21\end{array}$ & 5.08 & \\
\hline$(R)-9 \mathrm{~b}$ & 1.30 & 1.45 & 2.12 & 4.27 & 5.11 & \\
\hline$(R)-9 \mathrm{c}$ & 1.28 & 1.62 & & 4.27 & 5.38 & $\begin{array}{l}7.5(\mathrm{~m}, 3 \mathrm{H}, \mathrm{Ph}) \\
8.2(\mathrm{~m}, 2 \mathrm{H}, \mathrm{Ph})\end{array}$ \\
\hline$(R)-9 \mathrm{~d}$ & 1.32 & 1.60 & & 4.29 & 5.24 & $6.09\left(\mathrm{~s}, 1 \mathrm{H}, \mathrm{CHCl}_{2}\right)$ \\
\hline
\end{tabular}

a) Ausb. \% s. Tab. 3.

Chem. Ber. 119 (1986) 
$(R)-3$ beschrieben um. Die Verbindungen $(R)-9 \mathrm{a}, \mathrm{b}, \mathrm{c}$ werden destillativ gereinigt. Zur Darstellung von (R)-9d wird die etherische Lösung des Produkts nach Abfiltrieren des Unlöslichen im Rotationsverdampfer eingeengt, der Rückstand in $n$-Pentan aufgenommen und nach Abdestillieren des $n$-Pentans im Hochvak. getrocknet (Tab. 7).

(S)-2-(Acyloxy)propionsäure-ethylester (S)-9 aus (S)-Milchsäure-ethylester und Carbonsäurechloriden: Zur Darstellung der Verbindungen $(S)-9 \mathbf{a}, \mathbf{b}, \mathbf{c}$ tropft man unter Eiskühlung zu $5.91 \mathrm{~g} \mathrm{(50.0} \mathrm{mmol)} \mathrm{(S)-Milchsäure-ethylester} \mathrm{in} 10 \mathrm{ml}$ Chloroform und $10 \mathrm{ml}$ Pyridin die Lösung von jeweils $70 \mathrm{mmol}$ Carbonsäurechlorid (=5.49 g Acetylchlorid, $6.48 \mathrm{~g}$ Propionylchlorid, $9.84 \mathrm{~g}$ Benzoylchlorid) in $10 \mathrm{ml}$ Chloroform, rührt $8 \mathrm{~h}$ bei $20^{\circ} \mathrm{C}$, gießt das Gemisch auf Eis, säuert mit Salzsäure an und extrahiert mit Chloroform. Die organische Phase wird neutral gewaschen, getrocknet und destilliert. Ausb. $7.31 \mathrm{~g}(84 \%)(S)-9 \mathrm{a},[\alpha]_{\mathrm{D}}^{20}=$ $-44.5^{\circ}\left(c=5, \mathrm{CHCl}_{3}\right)$, Lit. $^{16)}(\mathrm{R})-9 \mathrm{a}:[\alpha]_{\mathrm{D}}^{20}=+41.9^{\circ}\left(c=5, \mathrm{CHCl}_{3}\right)$.

$6.07 \mathrm{~g}(76 \%)(S)-9 \mathrm{~b},[\alpha]_{\mathrm{D}}^{18}=-49.5^{\circ}$ (unverdünnt), Lit. ${ }^{17)}$ "l“-Form: $[\alpha]_{\mathrm{D}}^{18}=-47.63^{\circ}$, ,d"-Form: $[\alpha]_{D}^{22}=+51.54^{\circ}$.

$9.70 \mathrm{~g}(87 \%)(S)-9 \mathrm{c},[\alpha]_{\mathrm{D}}^{20}=+25.6^{\circ}$ (unverdünnt), Lit. ${ }^{17)},{ }^{\prime \prime}{ }^{\prime \prime}$-Form: $[\alpha]_{\mathrm{D}}^{20}=+24.56^{\circ}$, "l'-Form: $[\alpha]_{\mathrm{D}}^{20}=-24.6^{\circ}$.

(S)-9d wird analog dargestellt aus $3.54 \mathrm{~g}$ (30.0 mmol) (S)-Milchsäure-ethylester in $2.37 \mathrm{~g}$ ( $30.0 \mathrm{mmol})$ Pyridin und $20 \mathrm{ml}$ Dichlormethan mit $4.42 \mathrm{~g}$ (30.0 mmol) Dichloracetylchlorid in $20 \mathrm{ml}$ Dichlormethan. Nach Stehenlassen über Nacht bei Raumtemp. wird wäßrig aufgearbeitet, das Produkt in $n$-Pentan aufgenommen und nach Abdestillieren des $n$-Pentans der Rückstand i. Hochvak. destilliert. Ausb. $5.8 \mathrm{~g}(84 \%)$ farbloses $\mathrm{Ol},[\alpha]_{\mathrm{D}}^{20}=-33.6^{\circ}(c=5$, $\mathrm{CHCl}_{3}$ ).

Umsetzung von 2-(Methylsulfonyloxy)-(10b) bzw. 2-Brombernsteinsäure-diethylester (10d) mit Kaliumpropionat (8a'): Man rührt bei $20^{\circ} \mathrm{C} 0.28 \mathrm{~g}(2.5 \mathrm{mmol}) 8 \mathrm{a}^{\prime}$ mit jeweils $2.0 \mathrm{mmol}$ $10 \mathrm{~b}(0.537 \mathrm{~g})$ bzw. 10d $(0.506 \mathrm{~g})$ in $20 \mathrm{ml}$ Acetonitril und verfolgt die Umsetzung gaschromatographisch. Nach 1 h war weitgehende, nach 20 h vollständige Eliminierung zu Fumarbzw. Maleinsäure-diethylester (11) eingetreten und kein Substitutionsprodukt nachweisbar.

\section{(R)- bzw. (S)-2-(Propionyloxy)bernsteinsäure-diethylester [(R)-12a bzw. (S)-12a]}

a) Man läßt bei $-45^{\circ} \mathrm{C}$ zu $1.12 \mathrm{~g}(10.0 \mathrm{mmol})$ Kaliumpropionat (8a') in $40 \mathrm{ml}$ Acetonitril innerhalb $45 \mathrm{~min}$ die Lösung von $3.22 \mathrm{~g}$ (10.0 mmol) (S)-2-(Trifluormethylsulfonyloxy)bernsteinsäure-diethylester $[(S)-10 \mathrm{a}]^{2 \mathrm{a}}$ in $40 \mathrm{ml}$ Acetonitril tropfen, rührt $1.5 \mathrm{~h}$ bei $-45^{\circ} \mathrm{C}$ nach, läßt auf $20^{\circ} \mathrm{C}$ erwärmen und arbeitet wie bei $(R)-3$ beschrieben auf. Ausb. $1.27 \mathrm{~g}(52 \%)$ (R)-12a (nach Chromatographie über Kieselgel mit Dichlormethan), $[\alpha]_{\mathrm{D}}^{20}=+19^{\circ}(c=3.8$, $\mathrm{CHCl}_{3}$ ).

b) Man tropft bei $-65^{\circ} \mathrm{C}$ zu $0.925 \mathrm{~g}(10.0 \mathrm{mmol})$ Propionylchlorid in $25 \mathrm{ml}$ Dichlormethan langsam die Lösung von $1.9 \mathrm{~g}(10.0 \mathrm{mmol})(S)$-Äpfelsäure-diethylester und $0.79 \mathrm{~g}$ (10.0 mmol) Pyridin in $15 \mathrm{ml}$ Dichlormethan, rührt $1.5 \mathrm{~h}$ bei $-65^{\circ} \mathrm{C}$ nach, läßt auf $20^{\circ} \mathrm{C}$ erwärmen und noch ca. $2 \mathrm{~h}$ bei Raumtemp. stehen. Nach Waschen mit Wasser und Trocknen über Natriumsulfat wird über Kieselgel mit Dichlormethan chromatographiert. Ausb. $1.72 \mathrm{~g}$ $(70 \%)(S)-12 \mathrm{a},[\alpha]_{\mathrm{D}}^{20}=-19.2^{\circ}\left(c=4, \mathrm{CHCl}_{3}\right)$ - ${ }^{1} \mathrm{H}-\mathrm{NMR}\left(\mathrm{CDCl}_{3}\right): \delta=1.17(\mathrm{t}, 3 \mathrm{H}$, $\left.\mathrm{CH}_{3} \mathrm{CH}_{2}\right), 1.26\left(\mathrm{t}, J=7 \mathrm{~Hz}, 3 \mathrm{H}, \mathrm{CH}_{3} \mathrm{CH}_{2}\right), 1.28\left(\mathrm{t}, J=7 \mathrm{~Hz}, 3 \mathrm{H}, \mathrm{CH}_{3} \mathrm{CH}_{2}\right), 2.4(\mathrm{~m}, 2 \mathrm{H}$, $\mathrm{CH}_{3} \mathrm{CH}_{2}$ ), 2.87 (d, $\left.J=6 \mathrm{~Hz}, 2 \mathrm{H}, \mathrm{CH}_{2} \mathrm{CH}\right), 4.18\left(\mathrm{q}, J=7 \mathrm{~Hz}, 2 \mathrm{H}, \mathrm{CH}_{3} \mathrm{CH}_{2}\right), 4.22(\mathrm{q}, J=$ $7 \mathrm{~Hz}, 2 \mathrm{H}, \mathrm{CH}_{3} \mathrm{CH}_{2}$ ), 5.46 (t, $J=6 \mathrm{~Hz}, 1 \mathrm{H}, \mathrm{CH}$ ).

$$
\mathrm{C}_{11} \mathrm{H}_{18} \mathrm{O}_{6}(246.3) \text { Ber. C } 53.65 \text { H } 7.37 \text { (R)-12a: Gef. C } 53.57 \text { H } 7.50
$$

(S)-12a: Gef. C 53.91 H 7.63

(R)-2-(Benzoyloxy) bernsteinsäure-diethylester $[(R)-12 \mathrm{c}]$ : Wie bei $(R)-12 \mathrm{a}$ beschrieben aus $1.60 \mathrm{~g}$ (10.0 mmol) Kaliumbenzoat $(8 \mathrm{c})$ in $40 \mathrm{ml}$ Acetonitril und $3.22 \mathrm{~g}(10.0 \mathrm{mmol})(S)$ - 
$10 \mathrm{a}$ in $40 \mathrm{ml}$ Acetonitril. Ausb. nach Destillation $1.70 \mathrm{~g}(58 \%)$, Sdp. $132^{\circ} \mathrm{C} / 10^{-2}$ Torr (Lit. ${ }^{18)}$ $152^{\circ} \mathrm{C} / 0.5$ Torr, $l$-Form).

(R)-bzw. (S)-2-Acetoxy-3-phenylpropionsäure-methylester [(R)-bzw. (S)-16]

a) Wie bei $(R)-12 \mathrm{a}$ beschrieben aus $1.17 \mathrm{~g}(12.0 \mathrm{mmol})$ Kaliumacetat (8b') in $12 \mathrm{ml}$ Ace-

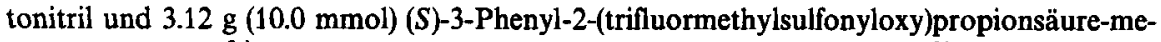
thylester $[(S)-15]^{2 \mathrm{a})}$ in $12 \mathrm{ml}$ Acetonitril. Ausb. $1.97 \mathrm{~g}(89 \%)(R)-16,[\alpha]_{\mathrm{D}}^{20}=+7.0^{\circ}(c=$ 4.8, $\mathrm{CHCl}_{3}$ ).

b) Man tropft unter Eiskühlung zu $3.60 \mathrm{~g}(20.0 \mathrm{mmol})(S)$-Phenylmilchsäure-methylester [(S)-14] (dargestellt durch azeotrope oder extraktive Veresterung von (S)-Phenylmilchsäure $[(S)-13],[\alpha]_{\mathrm{D}}^{25}=-26.9^{\circ}(c=2$, Aceton), mit Methanol/HCl $)$ in $10 \mathrm{ml}$ Chloroform und $3 \mathrm{ml}$ Pyridin die Lösung von $1.96 \mathrm{~g}(25.0 \mathrm{mmol})$ Acetylchlorid in $10 \mathrm{ml}$ Chloroform, rührt $2 \mathrm{~h}$ bei $20^{\circ} \mathrm{C}$ nach, gibt $50 \mathrm{ml}$ Chloroform zu, wäscht den Chloroformextrakt neutral, trocknet über Natriumsulfat und chromatographiert über Kieselgel mit Dichlormethan. Ausb. $4.17 \mathrm{~g}(94 \%)(S)-16,[\alpha]_{\mathrm{D}}^{20}=-7.0^{\circ}\left(c=4.8\right.$, Chloroform). $-{ }^{1} \mathrm{H}-\mathrm{NMR}\left(\mathrm{CDCl}_{3}\right): \delta=2.04$ (s, $3 \mathrm{H}, \mathrm{CH}_{3}$ ), 3.1 (m, $2 \mathrm{H}, \mathrm{CH}_{2}$ ), $3.68\left(\mathrm{~s}, 3 \mathrm{H}, \mathrm{CH}_{3}\right), 5.2$ (dd, $\left.1 \mathrm{H}, \mathrm{CH}\right), 7.2(\mathrm{~m}, 5 \mathrm{H}, \mathrm{Ph}$ ).

$$
\mathrm{C}_{12} \mathrm{H}_{14} \mathrm{O}_{4}(222.2) \text { Ber. C } 64.85 \text { H } 6.35 \text { Gef. C } 64.69 \text { H } 6.26
$$

(R)-bzw. (S)-Phenylmilchsäure [(R)-bzw. (S)-13]

a) $1.33 \mathrm{~g}(6.0 \mathrm{mmol})(R)-16$ werden mit $25 \mathrm{ml} 5$ proz. $\mathrm{KOH}$ in $25 \mathrm{ml}$ Methanol $3 \mathrm{~h}$ bei $20^{\circ} \mathrm{C}$ gerührt. Nach Ansäuern mit verd. Salzsäure wird im Rotationsverdampfer eingeengt, der Rückstand mit Aceton versetzt, die organische Lösung im Rotationsverdampfer eingeengt und der Rückstand i. Hochvak. getrocknet. Ausb. $0.94 \mathrm{~g}(94 \%)(R)-13$, Schmp. $121^{\circ} \mathrm{C}$, $[\alpha]_{\mathrm{D}}^{20}=+26.9^{\circ}(c=2$, Aceton).

b) Wie vorstehend aus $0.889 \mathrm{~g}(4.0 \mathrm{mmol})(S)-16$ und $15 \mathrm{ml} \mathrm{5proz}$. $\mathrm{KOH}$ in $15 \mathrm{ml} \mathrm{Me}$ thanol. Ausb. $0.609 \mathrm{~g}(92 \%)(S)-13$, Schmp. $125^{\circ} \mathrm{C},[\alpha]_{\mathrm{D}}^{25}=-27.0^{\circ}\left(c=2\right.$, Aceton). $-{ }^{1} \mathrm{H}$ NMR ([D $\left.\mathrm{D}_{6}\right]$ Aceton): $\delta=3.0\left(\mathrm{~m}, 2 \mathrm{H}, \mathrm{CH}_{2}\right), 4.47(\mathrm{dd}, 1 \mathrm{H}, \mathrm{CH}), 7.3(\mathrm{~m}, 3 \mathrm{H}, \mathrm{Ph}$ und $1 \mathrm{H}$, $\mathrm{OH})$.

Umsetzung von Benzolmethanthiol bzw. Thiophenolen 17: Man tropft bei 0 bzw. $-45^{\circ} \mathrm{C}$ (s. Tab. 4) zu den Schwefelverbindungen 17 und Kaliumcarbonat in Acetonitril die Lösung von (S)-1 in Acetonitril, rührt die angegebene Zeit bei der angegebenen Temp. nach (s. Tab. 4), engt im Rotationsverdampfer ein und arbeitet wie bei $(R)-3$ beschrieben auf (Tab. 8, 9).

2-(Thiocyanato) propionsäure-ethylester (19): Man tropft bei $0^{\circ} \mathrm{C}$ a) zu $0.972 \mathrm{~g}(10.0 \mathrm{mmol})$ Kaliumrhodanid in $20 \mathrm{ml}$ Acetonitril die Lösung von $2.5 \mathrm{~g}$ (10.0 mmol) 2-(Trifluormethylsulfonyloxy)propionsäure-ethylester (1 a) in $20 \mathrm{ml}$ Acetonitril bzw. b) zu $1.36 \mathrm{~g}(14.0 \mathrm{mmol})$ Kaliumrhodanid in $20 \mathrm{ml}$ Acetonitril die Lösung von $2.75 \mathrm{~g}(14.0 \mathrm{mmol})$ 2-(Methylsulfonyloxy)propionsäure-ethylester ( $1 \mathrm{~b}$ ) in $20 \mathrm{ml}$ Acetonitril, rührt bei a) $3 \mathrm{~h}$ bei $20^{\circ} \mathrm{C}$, bei b) $24 \mathrm{~h}$ bei $20^{\circ} \mathrm{C}$ und $3 \mathrm{~h}$ unter RückfluB $\left(82^{\circ} \mathrm{C}\right)$ und arbeitet wie bei $(R)-3$ auf. Die Kapillargaschromatographische Bestimmung von 2-(Isothiocyanato)propionsäure-ethylester (20) durch Koinjektion von 20 (siehe nachstehend) erfolgte vor der Destillation des Rohproduktes. - Ausb. a) $1.43 \mathrm{~g}(90 \%)$, b) $1.61 \mathrm{~g} \mathrm{(72 \% )} \mathrm{19,} \mathrm{Sdp.} 98^{\circ} \mathrm{C} / 12$ Torr, (Lit. $\left.{ }^{19)} 119^{\circ} \mathrm{C} / 20 \mathrm{Torr}\right)$. $-{ }^{1} \mathrm{H}$-NMR $\left(\mathrm{CDCl}_{3}\right): \delta=1.32\left(\mathrm{t}, J=7 \mathrm{~Hz}, 3 \mathrm{H}, \mathrm{CH}_{3}\right), 1.70\left(\mathrm{~d}, J=7 \mathrm{~Hz}, 3 \mathrm{H}, \mathrm{CH}_{3}\right), 3.97$ $(\mathrm{q}, J=7 \mathrm{~Hz}, 1 \mathrm{H}, \mathrm{CH}), 4.23\left(\mathrm{q}, J=7 \mathrm{~Hz}, 2 \mathrm{H}, \mathrm{CH}_{2}\right) .-{ }^{1} \mathrm{H}-\mathrm{NMR}\left(\mathrm{C}_{6} \mathrm{D}_{6}\right): \delta=0.90(\mathrm{t}, J=$ $\left.7 \mathrm{~Hz}, 3 \mathrm{H}, \mathrm{CH}_{3}\right), 1.25\left(\mathrm{~d}, J=7 \mathrm{~Hz}, 3 \mathrm{H}, \mathrm{CH}_{3}\right), 3.27(\mathrm{q}, J=7 \mathrm{~Hz}, 1 \mathrm{H}, \mathrm{CH}), 3.88(\mathrm{q}, J=$ $\left.7 \mathrm{~Hz}, 2 \mathrm{H}, \mathrm{CH}_{2}\right) .-{ }^{13} \mathrm{C}-\mathrm{NMR}\left(\mathrm{CDCl}_{3}\right): \delta=169.2(\mathrm{C}-1), 62.8\left(\mathrm{C}-1^{\prime}\right), 44.8(\mathrm{C}-2), 18.4(\mathrm{C}-3)$, $14.0\left(\mathrm{C}-2^{\prime}\right)$.

$$
\begin{array}{llllllll}
\mathrm{C}_{6} \mathrm{H}_{9} \mathrm{NO}_{2} \mathrm{~S}(159.2) & \text { Ber. } \mathrm{C} 45.27 & \mathrm{H} 5.70 & \mathrm{~N} 8.80 & \mathrm{~S} 20.14 \\
& \text { Gef. } \mathrm{C} 45.04 & \mathrm{H} 5.72 & \mathrm{~N} 8.93 & \mathrm{~S} 20.42
\end{array}
$$




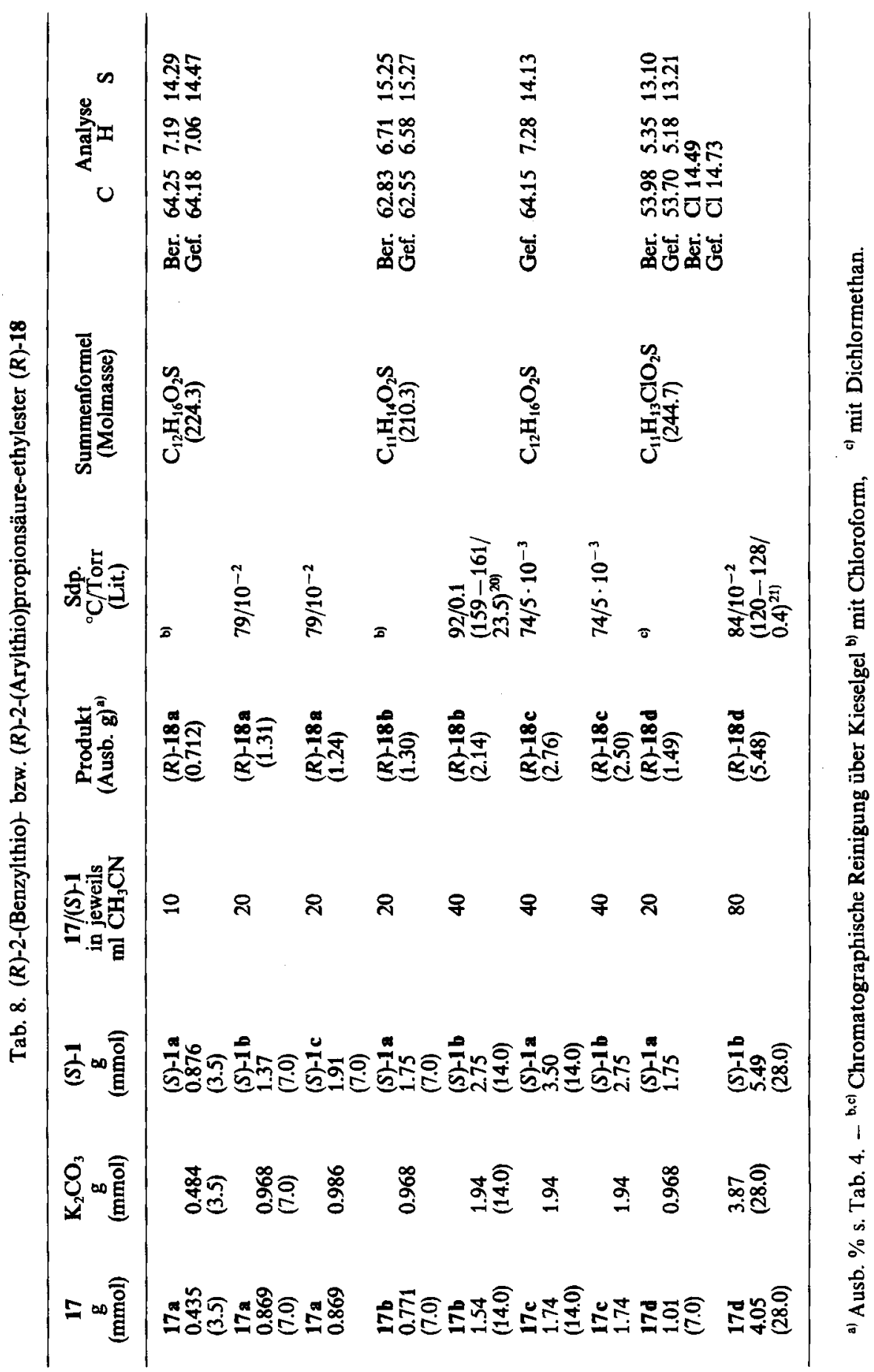

Chem. Ber. 119 (1986) 
Tab. 9. ${ }^{1} \mathrm{H}$-NMR-spektroskopische Daten von $(R)-18$ in $\mathrm{CDCl}_{3}(\delta, \mathrm{ppm})$

\begin{tabular}{cccccc}
\hline & $\begin{array}{c}3 \mathrm{H}, \mathrm{CH}_{3} \\
(\mathrm{t}, J=7 \mathrm{~Hz})\end{array}$ & $\begin{array}{c}1 \mathrm{H}, \mathrm{CH} \\
(\mathrm{q}, J=7 \mathrm{~Hz})\end{array}$ & $\begin{array}{c}3 \mathrm{H}, \mathrm{CH}_{3} \\
(\mathrm{~s})\end{array}$ & $\begin{array}{c}2 \mathrm{H}, \mathrm{CH}_{3} \mathrm{CH}_{2} \\
(\mathrm{q}, J=7 \mathrm{~Hz})\end{array}$ & $\begin{array}{c}\mathrm{Ph} \\
(\mathrm{m})\end{array}$ \\
\hline$(R)-18 \mathrm{a}$ & $\begin{array}{l}1.26 \\
1.36\end{array}$ & 3.25 & 3.79 & 4.12 & $7.2(5 \mathrm{H})$ \\
$(R)-18 \mathrm{~b}$ & 1.20 & 3.75 & & 4.16 & $7.4(5 \mathrm{H})$ \\
& 1.45 & & & & \\
$(R)-18 \mathrm{c}$ & 1.20 & 3.76 & 2.37 & 4.16 & $7.3(4 \mathrm{H})$ \\
$(R)-18 \mathrm{~d}$ & $\begin{array}{l}1.47 \\
1.20\end{array}$ & 3.77 & & 4.13 & $7.4(4 \mathrm{H})$ \\
& 1.47 & & & & \\
\hline
\end{tabular}

2-(Isothiocyanato ) propionsäure-ethylester (20), dargestellt nach Lit. ${ }^{12)} \mathrm{Sdp} .103^{\circ} \mathrm{C} / 25$ Torr (Lit. ${ }^{12)} 65^{\circ} \mathrm{C} / 5$ Torr). $-{ }^{1} \mathrm{H}-\mathrm{NMR}\left(\mathrm{CDCl}_{3}\right): \delta=1.32\left(\mathrm{t}, J=7 \mathrm{~Hz}, 3 \mathrm{H}, \mathrm{CH}_{3}\right), 1.60(\mathrm{~d}, J=$ $\left.7 \mathrm{~Hz}, 3 \mathrm{H}, \mathrm{CH}_{3}\right), 4.3\left(3 \mathrm{H}, \mathrm{CH}_{2} \mathrm{CH}\right) .-{ }^{1} \mathrm{H}-\mathrm{NMR}\left(\mathrm{C}_{6} \mathrm{D}_{6}\right): \delta=0.90\left(\mathrm{t}, J=7 \mathrm{~Hz}, 3 \mathrm{H}, \mathrm{CH}_{3}\right)$, $1.035\left(\mathrm{~d}, J=7 \mathrm{~Hz}, 3 \mathrm{H}, \mathrm{CH}_{3}\right), 3.65(\mathrm{q}, J=7 \mathrm{~Hz}, 1 \mathrm{H}, \mathrm{CH}), 3.84\left(\mathrm{q}, J=7 \mathrm{~Hz}, 2 \mathrm{H}, \mathrm{CH}_{2}\right)$. - ${ }^{13} \mathrm{C}-\mathrm{NMR}\left(\mathrm{CDCl}_{3}\right): \delta=169.0(\mathrm{C}-1), 62.5\left(\mathrm{C}-1^{\prime}\right), 54.9$ (C-2), 19.5 (C-3), $14.2\left(\mathrm{C}-2^{\prime}\right)$.

$\mathrm{C}_{6} \mathrm{H}_{9} \mathrm{NO}_{2} \mathrm{~S}$ (159.2) Gef. C 45.33 H 5.95 N 9.00 S 20.12

\section{CAS-Registry-Nummern}

(s)-1 a: 84028-88-6 / (s)-1 b: 63696-99-1 / (s)-1 c: $57057-80-4$ / ( \pm )-1 d: 41978-69-2 / 2a: 120 83-2 / 2b: 95-95-4 / 2c: 90-15-3 / 2d: 135-19-3 / (R)-3a: 66423-06-1 / (R)-3b: 66423-07-2 / (R)-3c: 57057-81-5/(R)-3d: $99967-48-3 /(R)-4$ a: $15165-67-0 /(R)-4 b: 30365-50-5 /(R)-4 c$ : 57128-29-7 / (R)-4d: 25153-08-6 / 5a: 120-80-9 / 5b: 108-46-3 / 5c: 123-31-9 / 5d: 87-66-1/ $(R, R)-6 \mathrm{a}: 99967-49-4 /( \pm)-6 \mathrm{a}: 100100-83-2$ / meso-6a: 100100-84-3/(R,R)-6b: $99967-$ 50-7/( \pm )-6b: 100100-85-4/meso-6b: 100100-86-5/(R,R)-6c: 99967-51-8/( \pm )-6c: 10010087-6 / meso-6c: $100100-88-7 /(R, R, R)-6 \mathrm{~d}: 99967-55-2 /(R, R)-7 \mathrm{a}: 99967-52-9 /(R, R)-7 \mathrm{~b}$ : 99967-53-0 / $(R, R)-7$ c: 99967-54-1 / 8a: 137-40-6 / 8a': 327-62-8 / 8a": 38869-24-8 / 8b': 127-08-2 / 8c': 582-25-2 / 8d': 19559-59-2 / (R)-9a: 99967-56-3 / (S)-9a: 99967-58-5 / (R)-9 b: 20918-92-7/ (S)-9b: 20918-91-6/(R)-9c: 835-86-9/(S)-9c: $1211-57-0 /(R)-9 \mathrm{~d}$ : 99967-57-4 / (S)-9d: $99967-59-6 /(S)-10$ a : 84028-87-5 / 10 b: $99967-60-9$ / 10d: 763-51-9 / (R)-12a: 99967-61-0 / (S)-12a: 99967-62-1 / (R)-12c: 99967-63-2 / (R)-13: 7326-19-4 / (S)-13: 20312-36-1 / (S)-14: 13673-95-5 / (S)-15: 84028-86-4 / (R)-16: $59246-58-1 /(S)-16$ : 30836-32-9 / 17 a: 100-53-8 / 17b: 108-98-5 / 17c: 106-45-6 / 17d: 106-54-7/(R)-18a: $99967-$ 64-3 / (R)-18b: 76685-16-0/(R)-18c: 99967-65-4/(R)-18d: 99967-66-5 / 19: 55602-05-6/ 20: 39574-16-8 / $\mathrm{CH}_{3} \mathrm{CH}(\mathrm{OH}) \mathrm{CO}_{2} \mathrm{Et}: \quad 687-47-8 / \mathrm{Cl}_{2} \mathrm{CHCOCl}: 79-36-7 /(E)-\mathrm{EtO}_{2} \mathrm{C}-$ $\mathrm{CH}=\mathrm{CHCO}_{2} \mathrm{Et}: \quad 623-91-6 /(Z)-\mathrm{EtO}_{2} \mathrm{CCH}=\mathrm{CHCO}_{2} \mathrm{Et}: \quad 141-05-9 / \quad(S)-\mathrm{EtO}_{2} \mathrm{CCH}_{2}$ $\mathrm{CH}(\mathrm{OH}) \mathrm{CO}_{2} \mathrm{Et}: 691-84-9$

1) U. Burkard, Dissertation, Univ. Stuttgart 1984.

2) 2a) F. Effenberger, U. Burkard und J. Willfahrt, Liebigs Ann. Chem. 1986, 314, dort weitere Literaturangaben. - ${ }^{26)} F$. Effenberger und $U$. Burkard, Liebigs Ann. Chem. 1986, 334.

3) 3a) C. H. Fawcett, R. L. Wain und F. Wightman, Ann. Appl. Biol. 43, 342 (1955). 3b) Compagnie Francaise de Produits Industriels (Erf. J. Schapira, J. Pecheur und R. Jacquier), D.O.S. 3001433 (17.7.1980) [Chem. Abstr. 94, 65307r (1981)].

4) 4a) $R$. Wegeler in Chemie der Pflanzenschutz- und Schädlingsbekämpfungsmittel, Bd. 8, Springer Verlag 1982 . - ${ }^{4 b)}$ Hoechst $A G$ (Erf. $H$. J. Nestler, G. Hörlein, $R$. Handte, $H$. Bieringer, F. Schwerdtle, P. Langelüddeke und P. Frisch), Europ. Patenschrift 0002800 B1 (2.12.1981) [Chem. Abstr. 95, 24528 r (1981)].

5) Sa) E. J. Corey, K. C. Nicolaou, M. Shibasaki, Y. Machida und C. S. Shiner, Tetrahedron Lett. 37, 3183 (1975). - ${ }^{\text {sb) }}$ O. Mitsunobu, Synthesis 1981, 1. 
6) 6a) W. H. Kruizinga, B. Strijtven und R. M. Kellogg, J. Org. Chem. 46, 4321 (1981). 6b) Nach Einreichen des vorliegenden Manuskripts erhielten wir Kenntnis von einer ebenfalls stereoselektiv verlaufenden Synthese von D- $\alpha$-Hydroxycarbonsäure-haltigen Dipeptiden aus L- $\alpha$-Halogencarbonsäureestern mit den Caesium-Salzen $N$-geschützter Aminosäuren (H.-G. Lerchen und H. Kunz, Tetrahedron Lett. 26, 5257 (1985)).

${ }^{7}$ G. Cainelli, F. Manescalchi, G. Martelli, M. Panunzio und L. Plessi, Tetrahedron Lett. 26, 3369 (1985).

8) 8a) N. Afza, A. Malik und W. Voelter, Z. Naturforsch., Teil B 39, $840(1984) .{ }^{8 \mathrm{~b})} R . W$. Binkley und D. J. Koholic, J. Carbohydr. Chem. 3, 85 (1984).

9) 9a) Santen Pharmaceutical Co. Ltd. (Erf. I. Mita, N. Toshioka und S. Yamamoto) US-Pat. 3246025 (12.4.1966) [Chem. Abstr. 65, $800 \mathrm{~g}(1966)]$. - ${ }^{96)}$ Mitsui Toatsu Chemicals, Inc. (Erf. K. Nitanai, A. Okubo und H. Tamazaki) Japan Kokai 7504092 (16.1.1975) [Chem. Abstr. 83, 114388 b (1975)].

10) G. Berse und G. Dupuis, Can. J. Chem. 43, 2174 (1965).

11) 11a) G. Claeson und J. Pedersen. Acta Chem. Scand. 22, 3155 (1968). - 11b) M. R. Detty und G. P. Wood, J. Org. Chem. 45, 80 (1980). - ${ }^{11 \mathrm{c})}$ S. Juliá, A. Ginebreda, J. Guixer und A. Tomás, Tetrahedron Lett. 21, 3709 (1980)

12) G. Losse und $H$. Weddige, Liebigs Ann. Chem. 636, 144 (1960).

13) M. Matell, Acta Chem. Scand. 16, 1065 (1962).

14) E. Gamstedt, Ark. Kemi 32, 151 (1970).

15) A. Fredga und M. Matell, Ark. Kemi 3, 429 (1951).

16) W. H. Kruizinga, B. Strijtveen und R. M. Kellogg, J. Org. Chem. 46, 4321 (1981).

17) J. Kenyon, H. Phillips und H. G. Turley, J. Chem. Soc. 1925, 399.

18) $K$. Freudenberg und $A$. Nö̈, Ber. Dtsch. Chem. Ges. 58, 2399 (1925).

19) W. Gerrard, J. Kenyon und $H$. Phillips, J. Chem. Soc. 1937, 153.

20) I. Minamida, Y. Ikeda, K. Uneyama, W. Tagaki und S. Oae, Tetrahedron 24, 5293 (1968).

21) S. L. Shapiro, I. M. Rose, F. C. Testa und L. Freedman, J. Am. Chem. Soc. 81, 5646 (1959).

$[232 / 85]$ 